\title{
Identification of microRNAs from Amur grape (vitis amurensis Rupr.) by deep sequencing and analysis of microRNA variations with bioinformatics
}

Chen Wang ${ }^{1}$, Jian Han ${ }^{1}$, Chonghuai Liư ${ }^{2}$, Korir Nicholas Kibet ${ }^{1}$ Emrul Kayesh${ }^{1}$, Lingfei Shangguan ${ }^{1}$, Xiaoying Li ${ }^{1}$ and Jinggui Fang ${ }^{1 *}$

\begin{abstract}
Background: MicroRNA (miRNA) is a class of functional non-coding small RNA with 19-25 nucleotides in length while Amur grape (Vitis amurensis Rupr.) is an important wild fruit crop with the strongest cold resistance among the Vitis species, is used as an excellent breeding parent for grapevine, and has elicited growing interest in wine production. To date, there is a relatively large number of grapevine miRNAs (Vv-miRNAs) from cultivated grapevine varieties such as Vitis vinifera $L$. and hybrids of $V$. vinifera and $V$. labrusca, but there is no report on miRNAs from Vitis amurensis Rupr, a wild grapevine species.

Results: A small RNA library from Amur grape was constructed and Solexa technology used to perform deep sequencing of the library followed by subsequent bioinformatics analysis to identify new miRNAs. In total, 126 conserved miRNAs belonging to 27 miRNA families were identified, and 34 known but non-conserved miRNAs were also found. Significantly, 72 new potential Amur grape-specific miRNAs were discovered. The sequences of these new potential va-miRNAs were further validated through miR-RACE, and accumulation of 18 new va-miRNAs in seven tissues of grapevines confirmed by real time RT-PCR (qRT-PCR) analysis. The expression levels of vamiRNAs in flowers and berries were found to be basically consistent in identity to those from deep sequenced sRNAs libraries of combined corresponding tissues. We also describe the conservation and variation of va-miRNAs using miR-SNPs and miR-LDs during plant evolution based on comparison of orthologous sequences, and further reveal that the number and sites of miR-SNP in diverse miRNA families exhibit distinct divergence. Finally, 346 target genes for the new miRNAs were predicted and they include a number of Amur grape stress tolerance genes and many genes regulating anthocyanin synthesis and sugar metabolism.

Conclusions: Deep sequencing of short RNAs from Amur grape flowers and berries identified 72 new potential miRNAs and 34 known but non-conserved miRNAs, indicating that specific miRNAs exist in Amur grape. These results show that a number of regulatory miRNAs exist in Amur grape and play an important role in Amur grape growth, development, and response to abiotic or biotic stress.
\end{abstract}

Keywords: Amur grape, microRNA, Sequences evolution, Solexa sequencing, miR-RACE, qRT-PCR

\footnotetext{
* Correspondence: fanggg@njau.edu.cn

'College of Horticulture, Nanjing Agricultural University, 1 Weigang, Nanjing

210095, China

Full list of author information is available at the end of the article
} 


\section{Background}

Endogenous small RNAs (sRNA) are important regulatory molecules with 19-25 nucleotides (nt) in length [1]. sRNAs can significantly regulate target gene expression levels at transcription or post-transcription levels by guiding mRNA degradation or translational repression [2-5]. MicroRNAs (miRNAs) are one of the main types of non-coding sRNAs and are widespread in organisms, playing important roles in plant growth, development and response to environmental stimuli. Usually, their expression exhibits time-dependent and tissue-specific characteristics across species. An increasing number of studies shows that many miRNAs might be implicated in organ development, cell differentiation and proliferation, cell death and cell apoptosis [6-9].

Currently, hundreds of miRNAs have been identified both by computational and/or experimental approaches in many plants [10-19], with a large number of the identified miRNAs being reported as conserved across various plant species. In addition, quite a number of species-specific miRNAs (young miRNAs), that can only be found in one or several plant species and have more evolution in their sequences than conserved (ancient) miRNAs have been identified. Usually, it is not easy to identify young miRNAs by traditional Sanger sequencing since they accumulate at lower levels in plants and comprise a much smaller fraction in sRNA libraries compared to the other abundant sRNAs. Recent advances in high-throughput sequencing technologies have however contributed significantly to the identification of lowly abundant non-conserved miRNAs in plants, with deep sequencing being successfully employed in the identification of conserved and non-conserved miRNAs in various plant species, including two Vitis populations [20-24]. Despite these advances, there is no information available regarding miRNAs in wild grapevine.

China is among the important centers of origin for plants in the Vitis genus and has abundant resources of wild grapevine, among which Amur grape has grown to be one of the most important wild fruit crops where it has been cultivated for wine, jam and as a table grapevine. In addition Amur grape is used as a valuable breeding parent for cold tolerance and resistance to devastating grape diseases like downy mildew, powdery mildew and anthracnose. Initiation of molecular biology and genomic studies on Amur grape is therefore of great significance with the identification of miRNAs in Amur grape being an important aspect of research that, to the best of our knowledge, has not been reported. In this study, we first constructed an Amur grape small RNA library and employed high throughput sequencing technology (Solexa) to deep sequence the small RNA library for the identification of new Amur grape
miRNAs (va-miRNAs) involved in regulation of cold and/or disease-resistance genes, and berry quality genes. This study also aimed at elucidating evolutionary conservation of these miRNA in other plants and its findings could enrich genomics research on miRNA-based regulatory systems in wild grapevines.

\section{Results}

\section{Analysis of sequences from small RNA library}

To identify new as well as conserved miRNAs in Amur grape, a wild species in the Vitis genus, a cDNA library of small RNAs ranging in size from 15 to $30 \mathrm{nt}$ and constructed using pooled RNA isolated from inflorescences, flowers and berries at various stages of development was deeply sequenced by Solexa. A total of $19,692,474$ reads were obtained from the sequencing datasets. After removing adaptor sequences, and filtering out low quality reads, $18,902,700$ (95.99\%) clean reads that included 6,740,185 unique sequences were obtained. Among the clean reads, 8,706,269 (46.06\%) matched the grapevine genome, among which 1,030,053 were observed to be similar to known miRNAs, while the other sequences belonged to other types of RNAs, including non-coding RNAs, tRNAs, rRNAs, snRNAs or snoRNAs. The numbers and proportions of these diverse categories of short RNAs are as shown in Table 1. From our library datasets, it was also observed that these small RNAs had uneven distribution in length (Additional file 1), where the class containing sRNAs of 24 nt was the most abundant $(36.94 \%)$ followed by the 21, 22 and 23 nt sequence classes in that order. The small RNAs with 20-24 nt in size accounted for about $85.00 \%$ of the total sequences, which is consistent with the typical sizes of miRNAs from Dicer digestion products [25].

Table 1 Distribution of small RNAs in Amur grape

\begin{tabular}{lllll}
\hline Category & Unique & Percent (\%) & Redundant & Percent (\%) \\
\hline exon_antisense & 121,519 & 1.80 & 301,729 & 1.60 \\
exon_sense & 211,613 & 3.14 & 616,910 & 3.26 \\
intron_antisense & 239,906 & 3.56 & 447,068 & 2.37 \\
intron_sense & 410,965 & 6.10 & 880,478 & 4.66 \\
miRNA & 1,426 & 0.02 & $1,030,053$ & 5.45 \\
rRNA & 97,064 & 1.44 & $1,858,381$ & 9.83 \\
repeat & 524,801 & 7.79 & $1,094,074$ & 5.79 \\
siRNA & 283,866 & 4.21 & $2,927,927$ & 15.49 \\
snRNA & 5,704 & 0.08 & 25,039 & 0.13 \\
snoRNA & 3,790 & 0.06 & 12,647 & 0.07 \\
tRNA & 23,935 & 0.36 & 842,565 & 4.46 \\
unann & $4,815,596$ & $\mathbf{7 1 . 4 5}$ & $8,865,829$ & 46.90 \\
\hline Total & $\mathbf{6 7 4 0 1 8 5}$ & $\mathbf{1 0 0 . 0 0}$ & $\mathbf{1 8 9 0 2 7 0 0}$ & $\mathbf{1 0 0 . 0 0}$ \\
\hline
\end{tabular}


Identification of conserved miRNAs in Amur grape

To identify the known miRNAs in Amur grape, we first compared the sequences from our sRNA library to known miRNAs from other plant species deposited in miRBase 16.0 http://www.mirbase.org/. Among the $18,902,700$ sequences screened, 1,030,053 unique sequences representing 126 conserved miRNAs were found to be orthologs of known miRNAs that had been earlier reported, and were conserved in Amur grape (Additional file 2). These identified va-miRNAs belong to 27 miRNA families and have been shown to be conserved in a variety of plant species according to a comparative genomics-based analysis [26]. Since deep sequencing may provide an alternative way to estimate the expression profiles of protein coding genes and/or miRNA genes [27,28], the millions of Amur grape sRNA sequences yielded by Solexa sequencing in this study can also allow us to estimate the abundance of various miRNA families and even that of different members in a given family [18]. This study shows that va-miRNA families display dramatic variations in copy number ranging from 2 to 372,442 (Additional file 2), indicating significant discrepancy of their accumulation in Amur grape. Eighty percent of these va-miRNAs have over 50 copies, with those having $>1,000$ copies accounting for about 50\%, while only four va-miRNAs (va-miR159a, vamiR159b, va-miR169i, va-miR171h) were sequenced less than 10 times (Figure 1) which is consistent with previous reports $[18,27,29]$ that the most conserved miRNAs are highly expressed in organisms.

To further identify the sequence divergences of orthologous miRNAs from diverse species of vitis plants, we compared conserved orthologous miRNAs between Amur grape and wine grapevine (released in miRbase 16.0) [20,21]. Results show that 38 unique conserved miRNA sequences of exhibited 1-3 nucleotide divergence at both ends of the sequence (Table 2). This phenomenon was also found in the alignment analysis between orthologous miRNAs from Amur grape and table grapevine [23], in which 12 unique orthologous sequences were observed to have 1-3 termini base divergences (Table 3 ). These sequence variations may reflect some evolution events that happened to the miRNAs in grapevine along with the speciation of the species, consistent with the reports in Arabidopsis thaliana and Arabidopsis lyrata [13,30]. Reverse transcription, amplification or sequencing could also be a cause of some of the nucleotide divergence observed [31].

\section{Identification of non-conserved va-miRNAs}

Among critical features that can be used to distinguish miRNAs from other small RNAs is the ability of a miRNA's flanking sequences to fold-back in a hairpin structure [32]. Based on the available grapevine genome sequence, we identified flanking sequences of the candidate va-miRNAs and predicted their possibility of forming characteristic hairpin structures (Additional file 3). Our analysis revealed that among the small RNA sequences matching the grapevine genome in these datasets, there were 3,537,694 sequences without any annotation, and whose flanking regions could be subjected to secondary structure analysis for prediction of new miRNA candidates. Our search for these new potential miRNAs revealed that 128,262 sequences, belonging to 106 unique sequences, were able to meet the new criteria of miRNA annotation set by Meyers et al. [32] and were thus considered as potential new vamiRNAs (Table 4). These newly identified va-miRNAs were 20-23 nucleotides long, and the negative folding free energies of their precursors ranged from $-20.8 \mathrm{kcal} /$ $\mathrm{mol}$ to $-124.6 \mathrm{kcal} / \mathrm{mol}$ except for va-miR066 and vamiR082 with $-19.7 \mathrm{kcal} / \mathrm{mol}$, and the average folding free energies of all these precursors were about -46.45 $\mathrm{kcal} / \mathrm{mol}$, which is similar to those of other plant miRNA precursors [33]. Although va-miR066 and vamiR082 have slightly higher free energies over $-20 \mathrm{kcal} /$ mol, one of the criteria of miRNAs annotation, our miR-RACE PCR could validate their real identity in Amur grape (Additional file 4). In addition, the precursor sequences for these 106 va-miRNAs can be mapped to the grapevine genome, whereby 71 match perfectly to grape ESTs datasets (Table 4). The precursors of these

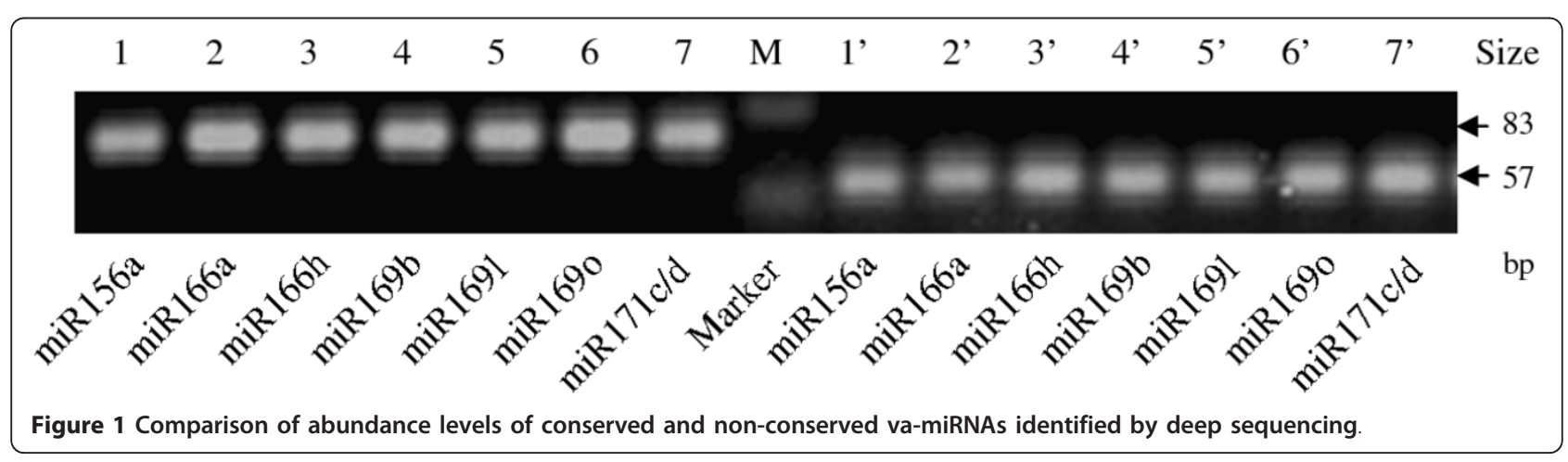


Table 2 List of miRNAs with nucleotide variations between Vitis amurensis Rupr. and Vitis vinifera

\begin{tabular}{|c|c|c|}
\hline MiRNA & Vitis amurensis Rupr. & MiRbase 16.0 (Vitis vinifera) \\
\hline MiR156a/i & TTGACAGAAGAGAGGGAGCAC & TGACAGAAGAGAGGGAGCAC \\
\hline MiR159a/b & CCTTGGAGTGAAGGGAGCT & CTTGGAGTGAAGGGAGCTCTC \\
\hline MiR166a & TCTCGGACCAGGCTTCATTCC & TCGGACCAGGCTTCATTCCTG \\
\hline MiR166c/e/h & TCGGACCAGGCTTCATTCCCC & TCGGACCAGGCTTCATTCCCCC \\
\hline MiR166d/f/g & TCGGACCAGGCTTCATTCCCC & TCGGACCAGGCTTCATTCCCCT \\
\hline MiR167a & TGAAGCTGCCAGCATGATCT & TGAAGCTGCCAGCATGATCTG \\
\hline MiR167b/e & TGAAGCTGCCAGCATGATCTAA & TGAAGCTGCCAGCATGATCTA \\
\hline MiR169b/h & TGAGCCAAGGATGGCTTGCCGT & TGAGCCAAGGATGGCTTGCCG \\
\hline MiR169e & TAGCCAAGGATGACTTGCCT & TAGCCAAGGATGACTTGCCTG \\
\hline MiR169i & TGAGCCAAGGATGACTGGCCGT & GAGCCAAGGATGACTGGCCGT \\
\hline MiR169| & TGAGCCAAGGATGACTTGCCG & GAGCCAAGGATGACTTGCCGT \\
\hline $\mathrm{MiR} 169 \mathrm{~m} / \mathrm{p}$ & TGAGCCAAGGATGACTTGCCG & GAGCCAAGGATGACTTGCCGG \\
\hline MiR169n/q & TAGAGCCAAGGATGACTTGCCG & GAGCCAAGGATGACTTGCCGG \\
\hline MiR169o & TGAGCCAAGGATGACTTGCCG & GAGCCAAGGATGACTTGCCGC \\
\hline MiR169r & TGAGTCAAGGATGACTTGCCGA & TGAGTCAAGGATGACTTGCCG \\
\hline MiR169t & CGAGTCAAGGATGACTTGCCGA & CGAGTCAAGGATGACTTGCCG \\
\hline MiR169x & TAGCCAAGGATGACTTGCCT & TAGCCAAGGATGACTTGCCTA \\
\hline MiR171a & TGATTGAGCCGTGCCAATATC & TTGAGCCGTGCCAATATCATG \\
\hline MiR171b & TTGAGCCGCGTCAATATCTCC & TGATTGAGCCGCGTCAATATC \\
\hline MiR171c/d & TGATTGAGCCGTGCCAATATC & TTGAGCCGTGCCAATATCACG \\
\hline MiR171e & TTGAGCCGCGCCAATATCACT & TGATTGAGCCGCGCCAATATC \\
\hline MiR171h & TTGAGCCGCGCCAATATCCCG & TGGTTGAGCCGCGCCAATATC \\
\hline MiR172d & TGAGAATCTTGATGATGCTGC & AGAATCTTGATGATGCTGCAT \\
\hline MiR319b & TTGGACTGAAGGGAGCTCCCT & CTTGGACTGAAGGGAGCTCCC \\
\hline MiR319c/f & TGCTTGGACTGAAGGGAG & CTTGGACTGAAGGGAGCTCCC \\
\hline MiR319g & TTGGACTGAAGGGAGCTCCC & ATTGGACTGAAGGGAGCTCCC \\
\hline MiR393a/b & TCCAAAGGGATCGCATTGAT & TCCAAAGGGATCGCATTGATCC \\
\hline MiR396a & TTCCACAGCTTTCTTGAA & TTCCACAGCTTTCTTGAACTA \\
\hline MiR396b & TTCCACAGCTTTCTTGAA & TTCCACAGCTTTCTTGAACT \\
\hline MiR396c/d & TTCCACAGCTTCTTGAA & TTCCACAGCTITCTTGAACTG \\
\hline MiR398a & TTCTCAGGTCACCCCTITGGG & TGTGTTCTCAGGTCACCCCTT \\
\hline MiR399a & CAAAGGAGAATTGCCCTGTTA & TGCCAAAGGAGAATTGCCCTG \\
\hline MiR399d & AAAGGAGATTTGCTCGTGAAT & TGCCAAAGGAGATTTGCTCGT \\
\hline MiR399h & TGCCAAAGGAGAATTGCC & TGCCAAAGGAGAATTGCCCTG \\
\hline MiR403a/b/c/d/e/elf & TTAGATTCACGCACAAACT & TTAGATTCACGCACAAACTCG \\
\hline MiR482 & ТСТTТССТАТССТСССАТTCC & ССТАСТССТСССАTTCC \\
\hline MiR535a/b/c/d/e & TGACAACGAGAGAGAGCACGC & TGACAACGAGAGAGAGCACGCT \\
\hline
\end{tabular}

conserved miRNAs were 65-311 nt long, where $66.1 \%$ were 65-150nt long, a finding similar to that observed in Arabidopsis and rice [34]. More importantly, out of these 106 va-miRNA sequences, 55 start with a 5 ' uridine, which is also an important characteristic feature of miRNAs. Considering that anti-sense miRNA (miRNA*) sequences are also an important criterion for identification of miRNAs $[27,28,33,35]$, we also searched for miRNAs* in our datasets where a total of 31 miRNAs* for new va-miRNAs candidates were found, providing evidence supporting them as new miRNAs. The remaining predicted new miRNAs without corresponding supporting miRNA* found can be considered as potential new
miRNAs, which can be due to that the level of corresponding miRNAs" were to low to be detected easily. As reported that the average frequency of miRNA* were established to be about $10 \%$ of that of mature miRNA [27], since quite a number of potential new miRNAs in our library had fewer than 10 reads observed, their corresponding miRNAs* could also be of a much lower frequency hence not easily to be sequenced. All characteristics of the predicted miRNAs mentioned above could indicate that they were potential new miRNAs in Amur grape. To further confirm whether or not these new miRNAs were Amur grape-specific, we compared them with those identified and reported in other 
Table 3 List of orthologous conserved miRNAs with nucleotide divergence between Amur grape and 'Summer Black' grape (hybrid of $\boldsymbol{V}$. vinifera and $\boldsymbol{V}$. labrusca)

\begin{tabular}{|c|c|c|}
\hline MiRNA & Amur grape & Summer Black grape \\
\hline $\mathrm{a} / \mathrm{b}$ & AAGGGAGCT & JAGCTCTC \\
\hline $67 a$ & TGA & TGAAGCTGCC \\
\hline iR169e & TAGCCAAGGATGACTTGCCT & TAGCC \\
\hline MiR169u & TGAGTCAAGGATGACTTGCCG & TGAGTCAAGGA \\
\hline ¡R319b & GGAGCTCCCT & TTGGACTG \\
\hline iR319c/f & TGCT & CC \\
\hline MiR393a & TCCAAAGGGATCGCATTGAT & GATCGCATTGAT \\
\hline MiR397a/b & TCATTGAGTGCAGCGTTGATG & CATTGAGTGCAGCGTTGATGA \\
\hline MiR398a & TTCTCAGGTCACCCCTTGGG & TGTGTTCTCAGGTCACCCCTT \\
\hline MiR399a & CAAAGGAGAATTGCCCTGTTA & TGCCAAAGGAGAATTGCC \\
\hline MiR399b/c & TGCCAAAGGAGAGTTGCCCTG & GCCAAAGGAGAGTTGCCCT \\
\hline MiR399d & AAAGGAGATTTGCTCGTGAAT & TCTGCCAAAGGAGATTTGCTC \\
\hline MiR399i & CGCCAAAGGAGAGTTGCCCTG & CGCCAAAGGAGAGTTGCCC \\
\hline
\end{tabular}

wine and table grapevine cultivars [20-24]. Results show that 72 out of the 106 newly identified miRNAs were discovered for the first time and thus might be Amur grape-specific; while the remaining 34 were found to also exist in the other vitis and a few other non vitis plant species (Table 4).

In addition, it was revealed that the read number for a majority of these new va-miRNAs were much lower than those for the conserved miRNAs (Figure 2), consistent with previous conclusions [10,13-16], indicating that new non-conserved miRNAs are usually expressed at lower levels and have some species-specific regulatory roles during growth and development of Amur grape.

\section{Validation of Amur grape miRNAs}

The abundance of different miRNAs in an organism varies with the levels of expression, which could influence their read numbers during sequencing. Theoretically, the higher the expression of miRNAs in organisms, the more the reads that can be sequenced and the higher the degree of accuracy in acquiring sequences during high throughput sequencing. This suggests the need for validation of the sequencing quality of new miRNAs having low expression levels. The obvious differences in the copies of miRNAs sequenced and the large number of low copy miRNAs in this study suggest the need for validation. This validation can be readily done using a specific PCR and PCR product sequencing, for which miR-RACE can be prefered method due to its effectiveness in the validation of precise sequences, especially both ends of miRNAs $[19,36]$. In this study, we performed validation of precise sequences of 20 low abundance va-miRNAs ( $<10$ reads) and 20 high abundance (> 100 reads) va-miRNAs selected randomly using miR-
RACE. The 20 low abundance va-miRNAs included 17 new and 3 conserved ones (e.g. va-miR398b, va-mir399c and va-miR828a), while the high abundance group included 10 conserved and 10 new va-miRNAs. miRRACE results demonstrate that all these 40 potential vamiRNA sequences could be clearly amplified in the 3'miR-RACE and 5'-miR-RACE analysis (Additional file 4). The miR-RACE products were cloned and sequenced for confirmation where identical results were generated by both high throughput sequencing and miR-RACE, thus further confirming the power of Solexa technology in sequencing sRNA libraries for miRNA discovery as well as ascertaining the existence of these miRNAs.

\section{Evolutionary conservation of miRNAs in Amur grape}

To study the conservation and evolutionary roles of these identified va-miRNAs, we conducted two aspects of comparative analysis for both conserved and new vamiRNAs. On one hand, extensive comparison between the identified va-miRNAs and the known miRNAs in 28 other plant species (including Vitis vinifera) from miRbase 16.0 were performed to investigate evolution of the identified conserved va-miRNAs (Table 5). On the other hand, all 106 va-miRNAs identified as potentialy new were used as query sequences to perform Blastn searches against all nucleotide sequences in NCBI databases for conservation analysis of these new miRNAs in a wide range of plant species.

Among the conserved miRNA sequences obtained from Amur grape, four miRNA families (va-miR403, vamiR477, va-miR479 and va-miR828) showed conservation in no more than five of the plant species investigated, with miR828 being found in only the Vitis and Arabidopsis genera, indicating that the four miRNA families have specificity in a narrow range of plants. As expected, Amur grape and Vitis vinifera shared 27 conserved miRNAs families, exhibiting higher conservation of miRNA families in these two grapevine species, which is also consistent with their closer genetic relationship. Nevertheless, vv-miR845 and vv-miR2111 in Vitis vinifera have no orthologs in Amur grape with vvmiR845 being unique to Vitis Vinifera, confirming the need for studies on Amur grape miRNAs for a comprehensive understanding of functions of miRNAs in vitis plants. We also discovered that apart from Vitis vinifera, va-miRNAs were much more conserved with their orthologs in poplar than those in the other species investigated, with a total of 25 miRNA families being conserved between Amur grape and poplar; while there were no orthologous miRNA families in Amur grape, Chlamydomonas reinhardtii and Vigna unguiculata. This phenomenon indicates that speciation of plants was accompanied with the specialization of miRNAs playing species-specific roles. 
Table 4 List of identified non-conserved miRNAs in Amur grape

\begin{tabular}{|c|c|c|c|c|c|c|c|c|c|}
\hline MiRNA ID & Sequences $\left(5 \rightarrow 3^{\prime}\right)$ & Reads & $\begin{array}{l}\text { Stem-loop length } \\
\text { (nt) }\end{array}$ & Start in Chr & MFE & $\begin{array}{l}\text { EST No. in Vitis } \\
\text { genus }\end{array}$ & $\begin{array}{l}\text { EST No. in other } \\
\text { plants }\end{array}$ & $\begin{array}{l}\text { MiRNAs homologs in other Vitis } \\
\text { species }\end{array}$ & miRNA* $^{*}$ \\
\hline va-miR001 ${ }^{\text {a }}$ & TITTITATTGGATCCGTCGGGA & 108 & 84 & 13:9154663 & -21.4 & $\begin{array}{l}\text { AM473732.1; } \\
\text { AM473732.1; }\end{array}$ & GU363535.1 & & \\
\hline va-miR002 & TTTGTIITTATTGTTGTIT & 14 & 240 & $16: 6817573$ & -42.05 & $\begin{array}{l}\text { AM432096.2; } \\
\text { AM436712.2 }\end{array}$ & & & \\
\hline va-miR003 & TITTCTTTTCCTGCATTITCT & 21 & 196 & 10_random:812676 & -28.77 & AM454974.2 & & & \\
\hline va-miR004 & TTTCCTATGATTTCTTGGCA & 18 & 139 & $17: 4715183$ & -44.23 & AM451502.1 & & & \\
\hline va-miR005 & TाTGCATGAGGGGGGATTGTCAT & 8 & 160 & $14: 10210052$ & -55.9 & AM425582.2 & & & \\
\hline $\begin{array}{l}\text { va-miR006 } \\
-1\end{array}$ & TाTCTIAGCAACCAAACAGAG & 10 & 213 & 3:3982162 & -36.6 & AM453475.2 & AC210553.1 & & \\
\hline $\begin{array}{l}\text { va-miR006 }{ }^{\mathrm{a}} \\
-2\end{array}$ & TाTCTTAGCAACCAAACAGAG & 8 & 213 & 3:3995013 & -36.6 & AM453475.2 & AC210553.1 & & \\
\hline $\begin{array}{l}\text { va-miR006 } \\
-3\end{array}$ & TाTCTAAGCAACCAAACAGAG & 8 & 255 & 19:4998280 & -74.2 & AM453475.2 & AC210553.1 & & \\
\hline va-miR007 b & TITCCGACTCGCACTCATGCCGT & 812 & 103 & 17:5737883 & -49 & No & & Vv-miR076 ${ }^{c}$ & Y \\
\hline va-miR008 ${ }^{a}$ & TTTCCACGGCTTTCTTGAACT & 16,273 & 182 & 1:1942578 & -57.1 & AM481354.2 & BT117687.1 & $\mathrm{V} v-\mathrm{miR} 075^{\mathrm{c}}$ & Y \\
\hline va-miR009 & TTTCCACATCTTTCTTGAACT & 10 & 113 & 12:7648535 & -49.5 & AM480580.2 & & & Y \\
\hline va-miR010 a & ПTTAATTAAATATTGAAGAT & 13 & 220 & 8:10844562 & -43.6 & no & $\begin{array}{l}\text { AC216916.1; } \\
\text { AC212928.1 }\end{array}$ & & \\
\hline va-miR011 ${ }^{\mathrm{b}}$ & TTGTCGCAGGAGAGACGGCACT & 6 & 92 & $14: 17492727$ & -57.8 & AM479856.1 & & $\operatorname{MirC} \sigma^{d}$ & Y \\
\hline va-miR012 & TTGGCACATTATCTAACAACT & 14 & 107 & 8:2707092 & -21.1 & No & & & \\
\hline va-miR013 & TTGCTGAGAGAGTCGTCTGCC & 53 & 81 & 9:13023036 & -38.5 & No & & & Y \\
\hline va-miR014 & TTGCTAAATCTTTGATTCGATC & 20 & 103 & 13:10216545 & -37.52 & AM443747.2 & & & \\
\hline va-miR015 & TTGCTAAATCATTGATTCGATC & 11 & 101 & 13:10213352 & -34.2 & $\begin{array}{l}\text { AM439308.2; } \\
\text { AM447569.2 }\end{array}$ & & & Y \\
\hline va-miR016 & TTCTTGTGATCTTGTTGTTC & 9,441 & 148 & $5: 20754701$ & -88.9 & AM454776.2 & & & \\
\hline va-miR017 & TTCTTCTTAACGTCTGACTTA & 10 & 135 & $1: 1002143$ & -57.6 & AM448689.1 & & & \\
\hline va-miR018 ${ }^{a}$ & TTCTCGGACCAGGCTTCATTC & 148 & 161 & $7: 14570131$ & -64.3 & no & BT089825.1 & Y & \\
\hline va-miR019 & TTCAAGTCAAAGTCGAACAAG & 16 & 71 & 1:15194533 & -20.2 & no & & & \\
\hline va-miR020 & TTATTAACCATTTAAATTTA & 5 & 212 & 4:14934239 & -21.55 & AM458370.2 & & & \\
\hline va-miR021 ${ }^{b}$ & TTATGTGAGTGTTCGGCAAATC & 16 & 90 & 5:20305533 & -37.9 & no & & $V_{v}-m i R 073^{c}$ & \\
\hline va-miR022-1 & TTAGTTGAACTAGGAGATGACA & 10 & 113 & 10:6246751 & -20.8 & no & & & \\
\hline va-miR022-2 & TTAGTTTGAACTAGGAGATGACA & 10 & 255 & $10: 6328614$ & -49.9 & no & & & \\
\hline va-miR023 b & TTAGATGATCATCAACAAACA & 9,128 & 118 & $5: 23154645$ & -45.5 & AM429664.2 & & 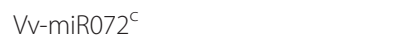 & Y \\
\hline va-miR024 & TTACTTITATCTGAATAGAAA & 8 & 108 & 15:3408926 & -26.7 & AM465770.2 & & & \\
\hline va-miR025 b & TTACACAGAGAGATGACGGTGG & 633 & 105 & 5:6660435 & -50.04 & AM429841.2 & & $\operatorname{MirC} 22^{d}$ & Y \\
\hline va-miR026 & TGGTAAATTGGTTTAAATATTC & 11 & 192 & 18:19596668 & -22.4 & $\begin{array}{l}\text { AM487707.2; } \\
\text { AM486523.2; } \\
\text { AM442872.2; } \\
\text { AM465537.1 }\end{array}$ & & & \\
\hline
\end{tabular}


Table 4 List of identified non-conserved miRNAs in Amur grape (Continued)

\begin{tabular}{|c|c|c|c|c|c|c|c|c|c|}
\hline va-miR027 a & TGGATGCATGTAGCTTGTCAA & 21 & 103 & 18:4024355 & -71.9 & AM484186.2; & $\begin{array}{l}\text { AK329204.1; } \\
\text { XM_002892267.1 }\end{array}$ & Y & \\
\hline va-miR028 & TGGATACACTTITTATTITIT & 6 & 204 & 10:2463091 & -29.5 & $\begin{array}{l}\text { AM467500.2; } \\
\text { AM425302.2; } \\
\text { AM476399.1 }\end{array}$ & & & \\
\hline $\begin{array}{l}\text { va-miR029b } \\
-1\end{array}$ & TGGAGAAGGGGAGCACGTGCA & 5 & 118 & 14:1404606 & -59 & AM462494.2; & & Vv-miR068c & \\
\hline $\begin{array}{l}\text { va-miR029b } \\
-2\end{array}$ & TGGAGAAGGGGAGCACGTGCA & 6 & 119 & $7: 3651397$ & -45.9 & AM462494.2; & & Vv-miR068c & \\
\hline va-miR030 & CGCCGCTCTCCTGTGACAAGA & 6 & 92 & 14:17492727 & -57.8 & AM479856.1 & & & \\
\hline va-miR031 ${ }^{\mathrm{b}}$ & TGCATTTGCACCTGCACCTTA & 104 & 165 & $6: 22063225$ & -75.22 & No & & Vv-miR020*c & Y \\
\hline va-miR032 & TGATTGTAGAAAATGTITITAAC & 5 & 111 & 6:21035897 & -36.7 & AM439539.1 & & & \\
\hline va-miR033 & TGATATTAGCAGCTGAGAACA & 10 & 147 & 14:11536480 & -70 & AM449587.2 & & & Y \\
\hline va-miR034 ${ }^{\text {b }}$ & TGAGTAGTGGACTATCGCATG & 9 & 116 & $17: 7362816$ & -43.9 & $\begin{array}{l}\text { AM489347.2; } \\
\text { AM461823.2; }\end{array}$ & Vv-miR065 & & \\
\hline va-miR035 & TGAGGAAGGGTGTTAGAGTAC & 13 & 90 & 13:5595054 & -46.1 & No & & & \\
\hline va-miR036 ${ }^{b}$ & TGACCGGCTCTTATCTCTCATG & 27 & 189 & 17:351716 & -79.3 & AM447150.1 & & Vv-miR063c & Y \\
\hline va-miR037 & TGACACTATATAAATATGAA & 6 & 244 & 2:8067706 & -48.1 & $\begin{array}{l}\text { AM430446.2; } \\
\text { AM466868.2; }\end{array}$ & & & \\
\hline va-miR038 & TGAATGGTTGGTAAATTGAG & 11 & 150 & 12:14954776 & -40.6 & $\begin{array}{l}\text { AM482866.2; } \\
\text { AM466422.2; }\end{array}$ & & & \\
\hline va-miR039 b & TGAAATGTAGGCAAGGAAAAG & 9 & 192 & $16: 8071148$ & -53.6 & AM478700.2; & & Vv-miR059c & \\
\hline va-miR040 & TCTGTTTGGACGCCGAGAAAA & 7 & 217 & 17:10557309 & -62.22 & No & & & \\
\hline va-miR041 ${ }^{b}$ & TCTGTCGCAGGAGAGATGATGC & 30 & 103 & 14:17809215 & -53.9 & No & & $\operatorname{MirC} 23^{d}$ & Y \\
\hline va-miR042 & TCTGTACTTTAAGAATCTGGCTT & 15 & 208 & 15:6195235 & -41.2 & AM454626.2 & & & Y \\
\hline va-miR043 & TCTGCATTTGCACCTGCACCT & 15 & 165 & 8:17999572 & -57.1 & No & & & \\
\hline va-miR044 & TCTCTTGATATTAGTAGCTGA & 9 & 138 & 14:11536485 & -71.9 & No & & & \\
\hline va-miR045 & TCGAGTGGGAACGCATTGAGC & 6 & 150 & 12:13192937 & -35.7 & AM486659.2 & & & \\
\hline va-miR046 ${ }^{b}$ & TCCCAGGAGAGATGGCACCTGC & 330 & 85 & 17:5984990 & -43 & AM457000.2 & & Vv-miR055 c & \\
\hline va-miR047 ${ }^{a}$ & TCACAAGTTCATCCAAGCACCA & 32,758 & 136 & 18_random:5237347 & -51.87 & No & EZ339498.1 & Vv-miR054 ${ }^{c}$ & Y \\
\hline va-miR048 & TCAATTAGTAGCTTAACATGG & 8 & 132 & 11:5115765 & -58.49 & AM471165.2 & & & \\
\hline va-miR049 & TCAATAAGGTACTITTAGCT & 108 & 126 & 11:12939237 & -34.3 & AM487033.2 & & Vv-miR053c & Y \\
\hline va-miR050 & TCAACTTGATTTTTGGATGAG & 9 & 101 & $8: 15743876$ & -27.8 & AM460148.2 & & & \\
\hline va-miR051 & TCAAAAGAGAAAATGTGGATG & 46 & 100 & $6: 4462385$ & -35.9 & AM428913.2 & & & \\
\hline va-miR052 & TATTAAGTTCAAGTGAAAATT & 5 & 206 & 10_random:1196749 & -64.5 & AM473600.2 & & & \\
\hline va-miR053 & TAGTGATTTTAGGAAGCGTTT & 7 & 271 & $16: 432019$ & -45.26 & AM455590.2 & & & \\
\hline va-miR054 b & TAGGAAACGTTTCTACTCTTT & 20 & 311 & scaffold_1047:19353 & -59.7 & No & & Vv-miR051c & \\
\hline va-miR055 ${ }^{\mathrm{b}}$ & TAATCTGCATCCTGAGGTCTA & 48 & 107 & 8:14329491 & -44.9 & AM426746.2 & & Vv-miR048 ${ }^{c}$ & Y \\
\hline va-miR056-1 & TAAAATCACTATCAAACGAA & 15 & 133 & 10:1636145 & -21 & AM454228.2 & & & \\
\hline va-miR056-2 & TAAAATCACTATCAAACGAA & 13 & 134 & $11: 1222860$ & -21.2 & AM454228.2 & & & Y \\
\hline va-miR056-3 & TAAAATCACTATCAAACGAA & 12 & 132 & 14:9946665 & -25.85 & AM454228.2 & & & \\
\hline
\end{tabular}


Table 4 List of identified non-conserved miRNAs in Amur grape (Continued)

\begin{tabular}{|c|c|c|c|c|c|c|c|c|c|}
\hline va-miR056-4 & TAAAATCACTATCAAACGAA & 13 & 255 & 16_random:3940513 & -39.5 & AM454228.2 & & & \\
\hline va-miR056-5 & TAAAATCACTATCAAACGAA & 15 & 136 & 8:11389633 & -33.7 & AM454228.2 & & & \\
\hline $\begin{array}{l}\text { va-miR057 } \\
-1\end{array}$ & GTTGGAAGTCGGTGGGGGACC & 7,884 & 91 & 19:13455035 & -47.9 & No & & VV-miR047c & Y \\
\hline $\begin{array}{l}\text { va-miR057 } \\
-2\end{array}$ & GTTGGAAGTCGGTGGGGGACC & 7,884 & 92 & 19_random:737237 & -42.6 & No & & Vv-miR047 & Y \\
\hline va-miR058 & GTAGCATCATCAAGATTCACA & 14 & 108 & 6:21821956 & -46.5 & AM439514.2 & & & \\
\hline va-miR059 a & GGAATCTTGATGATGCTGCAT & 18 & 152 & 6:5961963 & -69.27 & No & $\begin{array}{l}\text { GQ905588.1; } \\
\text { GQ419177.2 }\end{array}$ & Vv-miR038*c & Y \\
\hline va-miR060 & GAACATCCAGATCATAGGTATGA & 9 & 276 & 13:6835040 & -49.2 & AM470549.2 & & & \\
\hline va-miR061 & CTTCAGTGAGTGTGGCGTCAACG & 8 & 155 & 1_random:4350301 & -56.26 & AM424902.2 & & & \\
\hline va-miR062 & CTATGTTATAGGATCTTGGAT & 315 & 99 & 10:1456140 & -51.8 & No & & & Y \\
\hline va-miR063 ${ }^{b}$ & CTAGGAAGCGTTITAAATATT & 6 & 99 & 8:910651 & -38.93 & AM439566.1 & & Vv-miR035 & \\
\hline va-miR064 & CTAAAATCATTGTCAAACGAG & 12 & 303 & scaffold_352:50742 & -54.8 & AM449536.2 & & & \\
\hline va-miR065-1 & CTAAAATCACTACCAAACGG & 10 & 99 & 1:15345941 & -23 & AM432849.2 & & & \\
\hline va-miR065-2 & CTAAAATCACTACCAAACGG & 9 & 129 & $7: 12853717$ & -24.2 & AM432849.2 & & & \\
\hline va-miR066 & CGTCTTTTAAATTITITTATC & 6 & 121 & 7:11538839 & -19.7 & No & & & \\
\hline va-miR067 & CGGGAGATGACTACTGGAAG & 8 & 106 & $17: 2382956$ & -60.1 & No & & & \\
\hline va-miR068 ${ }^{a}$ & CGCTATCCATCCTGAGTTTCA & 375 & 122 & 8:8483671 & -54.4 & No & $\begin{array}{l}\text { AC235430.1; } \\
\text { FM163968.1; } \\
\text { FM163967.1 }\end{array}$ & Y & \\
\hline va-miR069 & CGCCGCTCTCCTGTGACAAGA & 6 & 92 & 14:17492727 & -57.8 & AM479856.1 & & & \\
\hline va-miR070 & CCAAGAGGGTGGAGTTCAGA & 10 & 111 & 14:13763190 & -54.9 & No & & & \\
\hline va-miR071 & CATGTGCCCCTCTTCCCCATC & 5 & 168 & 8:8981156 & -60.21 & AM459087.2 & & & \\
\hline va-miR072 & CATGGGCGGTTTGGTAAGAGG & 26,555 & 117 & 1:3849422 & -46.2 & No & & & Y \\
\hline va-miR073 & CATGACAAAAGATACTTCATT & 19 & 95 & 6:9332049 & -64.1 & No & & & \\
\hline va-miR074 & CAGGTTTATTGTTITTAATT & 7 & 95 & $\begin{array}{l}\text { 1_random:3034469 } \\
+\end{array}$ & -22 & No & & & \\
\hline va-miR075 & CAGGAAAGTCAGGGAAGTGTA & 9 & 93 & 13:6656017 & -41.93 & No & & & Y \\
\hline va-miR076 & CAAGTGTGGGATTTTGGGTGGCT & 14 & 187 & 4:15148566 & -59.2 & AM475853.2 & & & \\
\hline va-miR077 & ATTTCATCATTATATAAAACG & 5 & 260 & 2:10655532 & -124.6 & No & & & \\
\hline va-miR078 & ATTGACATCGTCTAACATAA & 27 & 96 & 13:908030 & -37.2 & No & & & \\
\hline va-miR079 & ATTATTITITTCATGGGAA & 7 & 183 & $6: 4945482$ & -40.4 & AM435008.2 & & & \\
\hline va-miR080 & ATTATATGTGTGAGCCGTTCA & 39 & 97 & 19:13648253 & -23.2 & No & & & \\
\hline va-miR081 & ATCATGTAGATATTGTCCGCT & 21 & 216 & 18:11023560 & -77.4 & No & & & \\
\hline va-miR082 ${ }^{b}$ & ATATTATTAGGGAATTAAGT & 4 & 82 & 18:15863697 & -19.7 & AM482601.2 & & Vv-miR024 & \\
\hline va-miR083 & ATACCATGTGGAAAAGAGGAATC & 89 & 199 & $16: 2126243$ & -38.7 & AM430285.2 & & & \\
\hline va-miR084 & ATAAAACAGGGCATAATTAG & 90 & 162 & 8:3276798 & -27.3 & AM455775.2 & & Vv-miR022 ${ }^{c}$ & \\
\hline va-miR085-1 & AGGGAAGGTCCCGTTGAGTT & 8 & 87 & 3:4651805 & -34.8 & AM432941.2 & & & \\
\hline va-miR085-2 & AGGGAAGGTCCCGTTGAGTT & 11 & 80 & scaffold_389:33261 & -31 & AM432941.2 & & & \\
\hline
\end{tabular}


Table 4 List of identified non-conserved miRNAs in Amur grape (Continued)

\begin{tabular}{|c|c|c|c|c|c|c|c|c|c|}
\hline va-miR086 & AGAGCCAAATATACTCCGCGG & 19 & 83 & 9:402800 & -52.1 & AM471736.2 & & & \\
\hline $\begin{array}{l}\text { va-miR087 } \\
-1\end{array}$ & AGAATGTGTTTGATAGTAATT & 7 & 220 & 13:10609418 & -32.71 & $\begin{array}{l}\text { AM450684.2; } \\
\text { AM470125.2; } \\
\text { AM466462.2 }\end{array}$ & Vv-miR013c & & \\
\hline $\begin{array}{l}\text { va-miR087 } \\
-2\end{array}$ & AGAATGTGTTTGATAGTAATT & 7 & 152 & 3:9026582 & -32.9 & $\begin{array}{l}\text { AM450684.2; } \\
\text { AM470125.2; } \\
\text { AM466462.2 }\end{array}$ & Vv-miR013c & & \\
\hline $\begin{array}{l}\text { va-miR087 } \\
-3\end{array}$ & AGAATGTGTTTGATAGTAATT & 7 & 134 & $5: 8615567$ & -39.8 & $\begin{array}{l}\text { AM450684.2; } \\
\text { AM470125.2; } \\
\text { AM466462.2 }\end{array}$ & Vv-miR013c & & \\
\hline $\begin{array}{l}\text { va-miR087 } \\
-4\end{array}$ & AGAATGTGTTTGATAGTAATT & 7 & 99 & $7: 6125455$ & -19.4 & $\begin{array}{l}\text { AM450684.2; } \\
\text { AM470125.2; } \\
\text { AM466462.2 }\end{array}$ & Vv-miR013c & & \\
\hline va-miR088 ${ }^{\mathrm{a}}$ & AGAAGAGAGAGAGTACAGCTA & 27 & 259 & $5: 17354630$ & -65.6 & No & $\begin{array}{l}\text { AL606589.3; } \\
\text { AY946858.1 }\end{array}$ & $\mathrm{V} v$-miR012 & \\
\hline va-miR089 & AGAAGAACAAGTAGACTGAGC & 11 & 95 & 12:17274522 & -35.6 & AM457953.2 & & & \\
\hline va-miR090 & AGAAAACTGTTTTTAAAAACT & 25 & 209 & $3: 3268552$ & -33.5 & AM428594.2 & & & \\
\hline va-miR091 & AAGTGGTGCTGTCTAGGGTAT & 8 & 153 & 16_random:2298331 & -74.9 & AM461957.1 & & & \\
\hline va-miR092 & AAGGTTACGAAGAATGTGAG & 7 & 65 & $6: 20373849$ & -28.4 & AM449474.1 & & & Y \\
\hline va-miR093 b & AAGGGTTTCTCACAGAGTTTA & 40 & 135 & 18:1385784 & -66.9 & AM428484.2 & & Vv-miR079 ${ }^{c}$ & \\
\hline va-miR094 b & AACTAACATAACTAAAGTGAA & 21 & 78 & $18: 7824675$ & -25 & AM444284.2 & & Vv-miR004 & \\
\hline va-miR095 & AACAAATGCTTGATTAAATG & 7 & 185 & 16:3892094 & -46.34 & AM456738.2 & & & \\
\hline va-miR096 & AAATTTGATTTTATGGTATT & 14 & 277 & $1: 4778703$ & -52.3 & AM479127.2 & & & \\
\hline va-miR097 & AAATTGGCTCTGTAAATTTC & 14 & 137 & 18:3503665 & -77.6 & AM454446.1 & & & Y \\
\hline va-miR098 & AAATTGACCTATTTAATAACT & 6 & 225 & 13:6487398 & -33.8 & No & & & \\
\hline va-miR099-1 & AAATAGAACAAAACACAGCAA & 8 & 101 & $4: 15872785$ & -57.2 & AM424238.2 & & & \\
\hline va-miR099-2 & AAATAGAACAAAACACAGCAA & 8 & 101 & scaffold_477:40535 & -63.2 & AM424238.2 & & & \\
\hline va-miR100 & AAAGTGCATTTGATAGTGATTC & 5 & 142 & 19_random:164412 & -29.22 & AM481492.2 & & & Y \\
\hline va-miR101 & AAAAATGAAAATGGGAGTCGGT & 11 & 243 & 5:12357599 & -42.41 & AM466362.2 & & & \\
\hline va-miR102 b & AAAAAGTTTGTCAAATATTG & 15 & 97 & 19:13648253 & -23.2 & AM435471.2 & & $V_{V}$-miRO01 ${ }^{c}$ & \\
\hline va-miR103 & AAAAAATTCAAAGGGAAATC & 8 & 162 & $2: 2995078$ & -26.63 & AM473477.2 & & & \\
\hline va-miR104 b & GGAATGGATGGTTAGGAGAG & 2,136 & & $17: 5578270$ & -54.4 & AM458290.1 & & $\operatorname{MirC13}{ }^{d}$ & Y \\
\hline va-miR105 & TAAAAGATAGGGATCGGATGGAG & 6 & 192 & 14:15112983 & -59.2 & AM463372.2 & & & \\
\hline va-miR106 & GTTGGAAGTCGGTGGGGGACC & 4,330 & 91 & 19:13455035 & -47.9 & AM481325.2 & & & \\
\hline
\end{tabular}




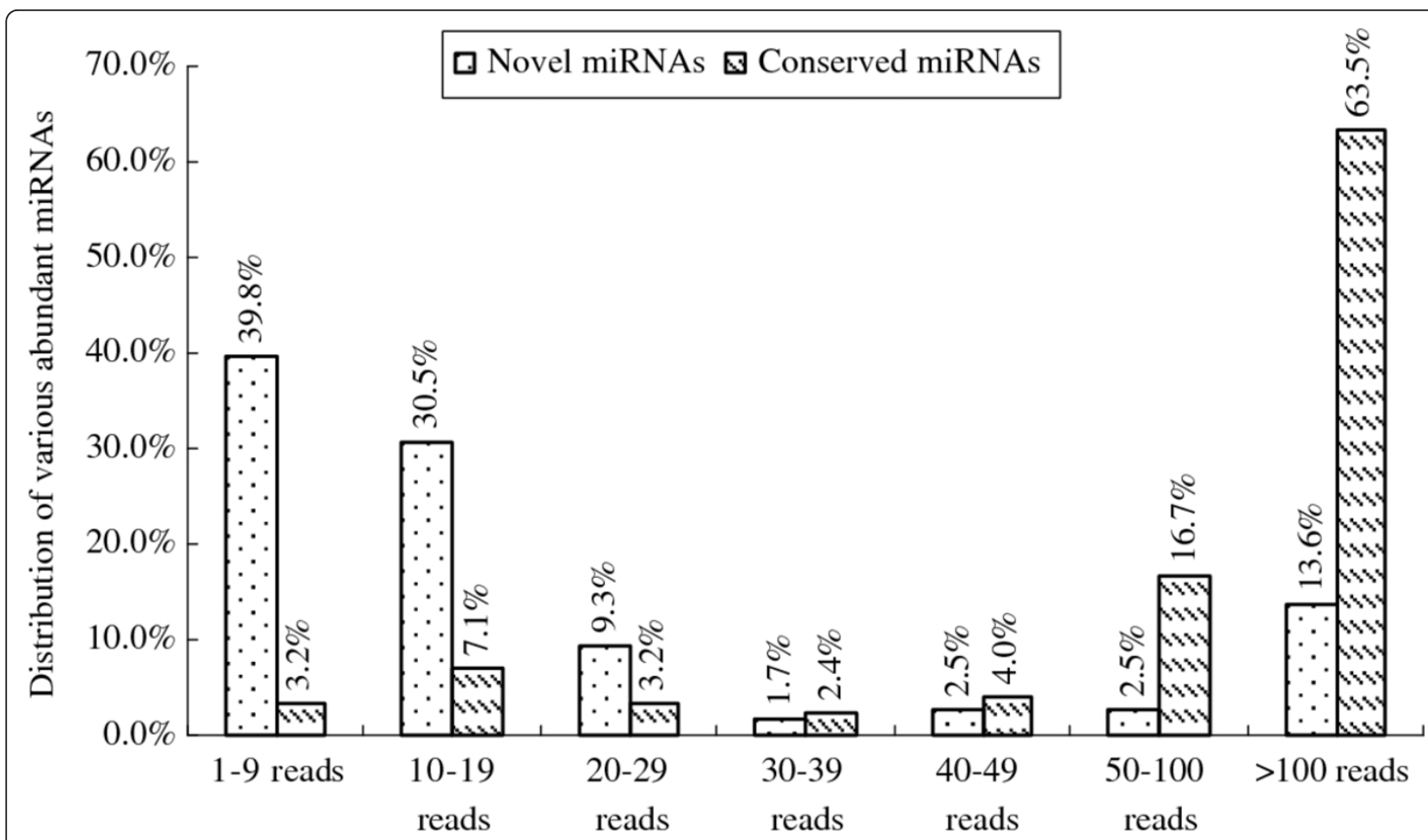

Figure 2 3'RACE and 5' RACE PCR products of seven amplified va-miR-SNPs shown in an ethidium bromide-stained agarose gel. Sizes of the molecular weight markers of the bottom and the top bands are $50 \mathrm{bp}$ and $100 \mathrm{bp}$, respectively. Lanes $1-7,1^{\prime}-7^{\prime}$ are $3^{\prime} \mathrm{RACE}$ (up) and 5'RACE (down) products of va-miR156a-SNP, va-miR166a-SNP, va-miR166h-SNP, va-miR169b-SNP, va-miR169l-SNP, va-miR169o-SNP and vamiR171c/d-SNP, respectively. The sizes of 3'RACE products are about 83 bp while the size of 5'RACE products are about 57 bp.

To further analyze the sequence evolutionary levels of 106 potential new va-miRNAs, these miRNAs were used as queries to search against the nucleotide sequence database in NCBI. The analysis results in Table 4 reveal that 96 of these va-miRNAs could not be found in any other plant species other than in Vitis vinifera, while only ten va-miRNAs (va-miR001, va-miR006, va-miR008, vamiR010, va-miR018, va-miR027, va-miR047, va-miR059, va-miR068 and va-miR088) have homologs in other plant species. Further comparison of the 96 va-miRNAs with $\mathrm{vv-miRNAs}$ identified from other wine and table grape cultivars showed that 72 va-miRNAs were exclusive to Amur grape, an indication that they could be Amur grape-specific and might possess some specific regulatory functions during Amur grape growth and development. The other 24 could be considered as vitis-specific due to their existence only in plants from the vitis genus.

\section{Analysis of va-miRNA SNP data from deep sequencing}

Since miRNAs are small functional units, single nucleotide shifts in the precursor elements as well as the mature miRNA sequences may drive the evolution of new miRNAs by altering their biological functions [37]. Furthermore, sequence variation around the processing sites, and sequence variations in the mature miRNA, particularly the seed sequence, may have profound affects on miRNA biogenesis and functions [37]. Uncovering and characterization of the single base substitution and length differences between wild type and variant sequences of conserved miRNAs can be quite helpful in the investigation of miRNA evolution within a plant species and/or across species. We reveal that out of the 126 conserved va-miRNAs identified in this study, 70 were only sequenced in their wild type sequences and their read numbers varied widely from 2 to 2337 , while the remaining 56 conserved miRNAs were detected in both the variant as well as wild type sequences, with copies of most of the former being much less than copies of the later. Similarly, we also identified a number of single base variants of miRNAs in the Fragaria vesca small RNA library (data not shown), indicating that this phenomenon may be not an occasional event derived from sequencing errors, miRNA SNPs might do exist in small RNA library. As reported in human liver miRNAs [38], these variants also included single nucleotide polymorphism (SNP) and/or length difference. To elucidate the characteristics of these miRNA variant phenomena, SNPs occurring in mature miRNA sequences were considered as miR-SNPs [37], while length differences of miRNAs were described 
Table 5 Analysis of orthologs of conserved miRNAs in 28 plant species

\begin{tabular}{|c|c|c|c|c|c|c|c|c|c|c|c|c|c|c|c|c|c|c|c|c|c|c|c|c|c|c|c|c|c|}
\hline $\begin{array}{l}\text { Plant } \\
\text { name }\end{array}$ & $\begin{array}{l}\text { miR1 } \\
56\end{array}$ & $\begin{array}{l}\text { miR1 } \\
59\end{array}$ & $\begin{array}{l}\text { miR1 } \\
60\end{array}$ & $\begin{array}{l}\text { miR1 } \\
62\end{array}$ & $\begin{array}{l}\text { miR1 } \\
64\end{array}$ & $\begin{array}{l}\text { miR1 } \\
66\end{array}$ & $\begin{array}{l}\text { miR1 } \\
67\end{array}$ & $\begin{array}{l}\text { miR1 } \\
68\end{array}$ & $\begin{array}{l}\text { miR1 } \\
69\end{array}$ & $\begin{array}{l}\text { miR1 } \\
71\end{array}$ & $\begin{array}{l}\text { miR1 } \\
72\end{array}$ & $\begin{array}{l}\text { miR3 } \\
19\end{array}$ & $\begin{array}{l}\text { miR3 } \\
90\end{array}$ & $\begin{array}{l}\text { miR3 } \\
93\end{array}$ & $\begin{array}{l}\text { miR3 } \\
94\end{array}$ & $\begin{array}{l}\text { miR3 } \\
95\end{array}$ & $\begin{array}{l}\text { miR3 } \\
96\end{array}$ & $\begin{array}{l}\text { miR3 } \\
97\end{array}$ & $\begin{array}{l}\text { miR3 } \\
98\end{array}$ & $\begin{array}{l}\text { miR3 } \\
99\end{array}$ & $\begin{array}{l}\text { miR4 } \\
03\end{array}$ & $\begin{array}{l}\text { miR4 } \\
08\end{array}$ & $\begin{array}{l}\text { miR4 } \\
77\end{array}$ & $\begin{array}{l}\text { miR4 } \\
79\end{array}$ & $\begin{array}{l}\text { miR4 } \\
82\end{array}$ & $\begin{array}{l}\text { miR5 } \\
35\end{array}$ & $\begin{array}{l}\text { miR8 } \\
28\end{array}$ & $\begin{array}{l}\text { miR8 } \\
45\end{array}$ & $\begin{array}{l}\mathrm{miR} \\
2111\end{array}$ \\
\hline P01 & + & + & + & + & + & + & + & + & + & + & + & + & + & + & + & + & + & + & + & + & + & + & + & + & + & + & + & - & - \\
\hline P02 & + & + & + & + & + & + & + & + & + & + & + & + & + & + & + & + & + & + & + & + & + & + & + & + & + & + & + & + & + \\
\hline P03 & + & + & + & + & + & + & + & + & + & + & + & + & + & + & + & + & + & + & + & + & + & + & - & - & - & - & + & - & + \\
\hline P04 & + & + & + & - & + & + & + & + & + & + & + & + & + & + & + & + & + & + & + & + & - & + & - & - & - & + & - & - & - \\
\hline P05 & + & + & + & - & + & + & + & + & + & + & + & + & + & + & + & + & + & - & - & + & - & + & - & - & + & - & - & - & - \\
\hline P06 & + & + & + & - & + & + & + & + & + & + & + & + & + & + & + & + & + & + & - & + & - & + & - & - & - & - & - & - & - \\
\hline P07 & + & + & + & + & + & + & + & + & + & + & + & + & + & + & - & + & + & - & + & + & - & - & - & - & - & - & - & - & + \\
\hline P08 & + & + & - & - & - & - & - & + & - & - & - & - & - & - & - & - & + & - & - & - & - & + & - & - & - & - & - & - & - \\
\hline
\end{tabular}

P10 +

P12

P13

P15 +

P16+

P17 +

P18

P21

P22

$\mathrm{P} 23$

P24

P25

P28

Notes: P01, Vitis amurensis Rupr.; P02, Vitis vinifera; P03, Arabidopsis thaliana; P04, Oryza sativa; P05, Zea mays; P06, Sorghum bicolor; P07, Medicago truncatula; P08, Saccharum spp.; P10, Glycine max; P10, Populus trichocarpa; P11, Physcomitrella patens; P12, Gossypium hirsutum; P13, Chlamydomonas reinhardtii; P14, Brassica napus; P15, Pinus taeda; P16, Selaginella moellendorffii; P17, Triticum aestivum; P18, Carica papaya; P19, Solanum lycopersicum; P20, Lotus japonicus; P21, Vigna unguiculata; P22, Malus domestica; P23, Phaseolus vulgaris; P24, Brachypodium distachyon; P25, Aquilegia caerulea; P26, Citrus sinensis; P27, Ricinus communis; P28, Arachis hypogaea; + with orthologs, - without orthologs. 
as miR-LDs. In total, 43 kinds of miR-LDs and 117 types of miR-SNPs were identified from 43 and 56 conserved va-miRNAs, respectively (Additional file 5 and Additional file 6). Even though the number of miR-SNP types is greater than that of miR-LDs, the read number of miR-SNP is lower than that of miR-LDs, and the read numbers of both are far lower than in the corresponding wild types. In order to further validate the existence of these variant phenomena, some sequences with variant bases were randomly selected and subjected to experimental verification. Here, miR-RACE was utilized to verify existence of ten miR-LDs, among which seven were successfully validated (Figure 1). These results to some extent confirm the reality and existence of variant phenomena (miR-SNPs, miR-LDs etc.) in sequences during miRNAs evolution.

It should be noted that only one miR-LD could be detected in each conserved va-miRNA, while one to five types of miR-SNPs were found in the same va-miRNA, suggesting that sequence variations in seed sequences of miRNAs may be among important factors driving the evolution of new miRNAs, even though their abundance is much lower than that of wild types. The numbers of miR-SNPs located in different members of the same miRNA family and those in diverse miRNA families were all distinctly different. Although the va-miR169 family had the highest number of miRNA members with SNPs, the va-miR166 family topped in the list of total number of SNPs, followed by va-miR156, va-miR167 and va-miR169 families (Figure 3). The number of SNPs in each member of the other miRNA families was lower than six. These situations suggest potential divergence in conservation during evolution of va-miRNAs. In addition, SNP sites in different members of some va-miRNA families were identical or quite similar, which can be attributed to all members of each group targeting identical or similar genes, making diverse members of the same miRNA family exhibit functional conservation. The best example of this is the va-miR160 family, where nucleotide variants of all its six members (a, b, c, d, e, f) not only exist in the middle of their mature sequences, but all have an identical miRNA SNP site. Since this site was located at a miRNA-mRNA mismatch position as well as at a discrepancy site in diverse members (Figure 4), this miR-SNP in the va-miR160 family is unlikely to change the target genes of these miRNAs. The SNP sites of different members in other va-miRNA families were clearly divergent, with most of the SNPs located in mature va-miRNAs sequences seeming to increase the mismatch positions of miRNA-mRNA binding which could result in changes in their targeting patterns or losing target roles, and also become a potential cause for generation of new miRNAs. The best example of this phenomenon is the location of SNPs in the vamiR156 family, where 15 of the 20 SNPs created new mismatch sites from the match position upon which miRNAs bind their target genes, while the variant bases of the other five SNPs occurred in the original mismatch positions of miRNAs and the binding regions in their target genes. This suggests that nucleotide

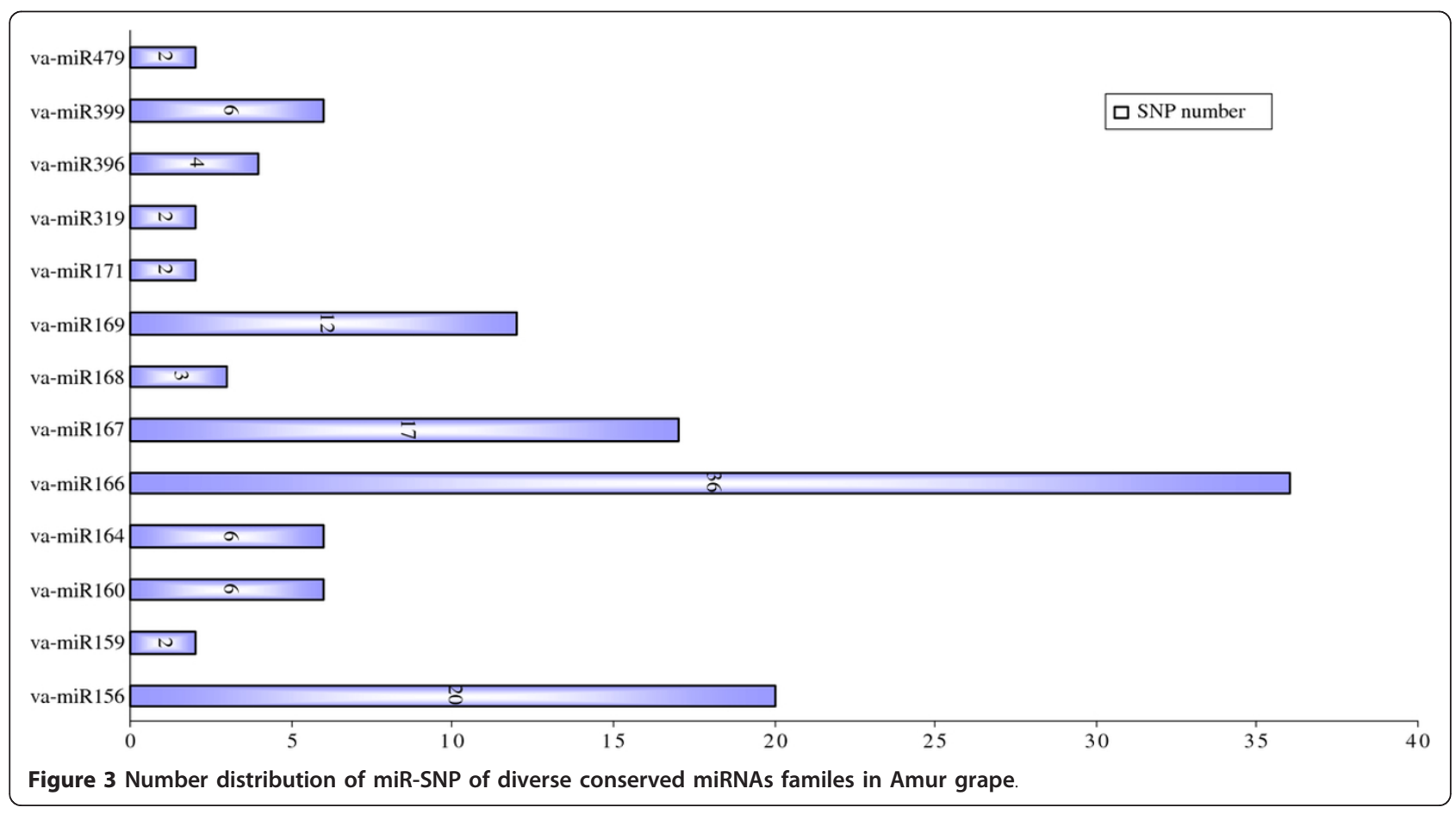




\section{Mismatch base sites 15 \\ AK373591.1 TGCCTGGCTCCCTGCATGCCA (Target gene) \|\|\|\|\|\|\|\|\|\|\|\|$\|$ Va-miR160a TGCCTGGCTCCCTGAATGCCA miR160a- SNP TGCCTGGCTCCCTGTATGCCA AK373591.1 TGCCTGGCTCCCTGCATGCCA (Target gene) \|\|\|\|\|\|\|\|\|\|\|\| Va-miR160b TGCCTGGCTCCCTGAATGCCA miR160b-SNP TGCCTGGCTCCCTGTATGCCA AK373591.1 TGCCTGGCTCCCTGCATGCCA (Target gene) \|\|\|\|\|\|\|\|\|\|\|\|$\|$ Va-miR160c TGCCTGGCTCCCTGTATGCCA miR160c-SNP TGCCTGGCTCCCTGAATGCCA AK373591.1 TGCCTGGCTCCCTGCATGCCA (Target gene) $\quad\|\|\|\|\|\|\|\|\|\|\|\| \|$ Va-miR160d TGCCTGGCTCCCTGTATGCCA miR160d-SNP TGCCTGGCTCCCTGAATGCCA AK373591.1 TGCCTGGCTCCCTGCATGCCA (Target gene) $\quad\|\|\|\|\|\|\|\|\|\|\|\| \|$ Va-miR160e TGCCTGGCTCCCTGAATGCCA miR160c-SNP TGCCTGGCTCCCTGTATGCCA AK373591.1 TGCCTGGCTCCCTGCATGCCA (Target gene) $|\|\|\|\|\|\|\|\||\|\| \|$ Va-miR160f TGCCTGGCTCCCTGTATGCCA miR160f-SNP TGCCTGGCTCCCTGAATGCCA}

Figure 4 Comparison of polymorphisms of va-miR160a, b, c, d, e and AK373591.1 binding sites, respectively. Va-miR160a, vamiR160b, va-miR160c, va-miR160d, va-miR160e respectively showed wild type sequences; miR160a-SNP, miR160b-SNP, miR160c-SNP, miR160d-SNP, miR160e-SNP exhibited SNP types of these miRNAs.

variation within these miRNA families is likely to be one of the important driving powers in the evolution of new miRNAs, consistent with that reported in miRNAs of Arabidopsis and humans [37-39]. Compared to Arabidopsis and humans, these miR-SNPs were less abundant in Amur grape. The various characteristics of SNP sites from the two miRNA families (va-miR160 and vamiR156) above suggest that significant divergence exists in miRNA conservation and evolution among different miRNA families.

\section{Analysis of the expression profiles of va-miRNAs}

High throughput sequencing can provide an efficient method for the detection of expression profiles of miRNAs as well as the identification of miRNA sequences. Usually, highly expressed miRNAs can be sequenced at high rates and a large number of their reads can be generated. Results from Solexa sequencing exhibit drastically different expression levels of members of different miRNAs families [12,14,18,25,28]. Abundance comparison of similar or different miRNAs in various tissues could present valuable information for better understanding of the roles of miRNAs. Based on the diverse abundance of miRNAs in our Amur grape sRNAs library, three groups of new va-miRNAs were randomly selected and aptly named Groups I, II and III. Group I comprised highly abundant va-miRNAs with over 4,000 reads, Group II had moderately abundant va-miRNAs with 100-4,000 reads while Group III had lowly abundant va-miRNAs with $<100$ reads. To study spatiotemporal expression of the new va-miRNAs having diverse copies and obtained by deep sequencing of the mixed sRNA library of different organs and tissues, six va-miRNAs per group (Figure 5 ) were randomly selected and their expression profiles in young leaves, large leaves, stems, tendrils, flowers, young berries and large berries analyzed with real-time PCR (qRT-PCR). The qRT-PCR expression results (Figure 5) show that even though the qRT-PCR result of va-miRNAs in flowers and berries at different developing stages could not be compared well with those data from deep sequencing the combined sRNA libraries, totally, qRT-PCR expression levels of va-miRNAs from flowers and berries at various developing stages were relatively consistent with those shown as read numbers from deep sequencing sRNAs libraries of combined corresponding tissues, confirming that deep sequencing can accurately detect accumulation levels of miRNAs in organisms. As shown in Figure 5, the qRT-PCR expression profiles of va-miRNAs in young and large leaves, stems, tendrils, inflorescences, flowers and berries have significant differences and can thus demonstrate the existence of spatiotemporal specificity in miRNA expression levels, an observation that could provide important insight to functions of va-miRNAs. Further analysis on the expression modes of 18 va-miRNAs in the three groups reveals that 13 va-miRNAs were expressed in flower and/or berry tissues of Amur grape. Eight va-miRNAs were only expressed in single tissues investigated, but their accumulation levels exhibited clear differences as exemplified by va-miR025, va-miR059 and va-miR072 in flowers, vamiR023 and va-miR056 in young berries, va-miR056, vamiR057 and va-miR069 in large berries, and va-miR008, va-miR016, va-miR068 and va-miR097 in both young and large berries. One va-miRNA (va-miR031) was expressed only in reproductive organs (flowers and berries) (Figure 5). These observations indicate that expression characteristics of most new va-miRNAs in this study were flowerand/or berry-specific, an aspect attributed to our small RNA library coming from a mixture of flower and berry tissues. It was also observed that va-miR015, va-miR018 

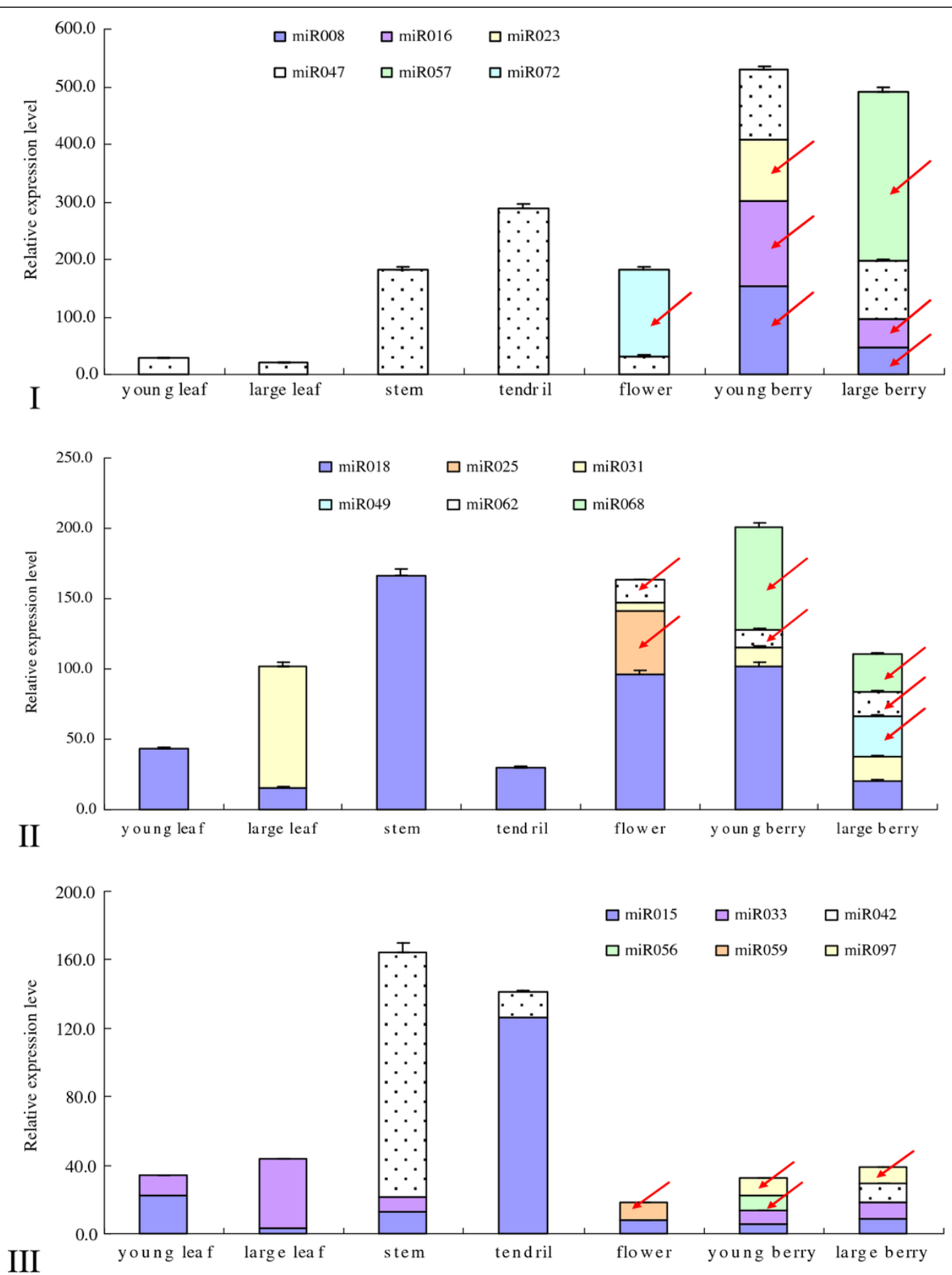

Figure 5 Real-time RT-PCR expression profiles of va-miRNAs with three frequencies from deep sequencing sRNA library in seven tissues of Amur grape. I, II, III denote va-miRNAs with high, moderate and low frequency in the deep sequenced sRNA library, respectively; the red arrows denote the flower- and/or berry-specific miRNAs. Lanes: young leaf $(4 \mathrm{~cm}$ in diameter), large leaf $(8 \mathrm{~cm}$ in diameter), stem $(0.2 \mathrm{~cm}$ in diameter), tendril $(0.1 \mathrm{~cm}$ in diameter), inflorescence $(0.2 \mathrm{~cm}$ in diameter per grain), flower $(0.25 \mathrm{~cm}$ in diameter per grain), young berry $(0.8 \mathrm{~cm}$ in diameter) and large berry $(1.5 \mathrm{~cm}$ in diameter). Each reaction was repeated three times and the template amount was corrected by $5.8 \mathrm{~s}$ rRNAs. 
and va-miR047 were ubiquitously expressed but at varying levels in all organs studied. These phenomena indicate that the extent/strength of miRNA regulatory roles during various development stages of Amur grape might have significant divergence. The other two va-miRNAs (va-miR033 and va-miR042) showed another kind of expression pattern where they were expressed in most but not all of the tissues investigated. All these findings point to typical spatiotemporal specificity of these new va-miRNAs. Generally, qRT-PCR expression profiles not only confirmed the existence of these new miRNAs in Amur grape, but also suggest that these va-miRNAs possess tissue- or developmental stages-specificity, which can in turn lay a solid foundation for studies on detailed functions of va-miRNAs.

\section{Prediction of va-miRNA target genes}

The prediction of miRNA target genes is important in comprehensive studies of miRNA gene-regulation functions. Currently, bioinformatics methods based on the high homology between miRNAs and target genes are considered one of the most efficient approaches used to verify target genes, and has been confirmed in quite a number of studies [40-43]. To better understand the functions of va-miRNAs, their putative targets were predicted using a BLASTN algorithm based on the homology between miRNAs and their target genes in grapevines following the rules of target prediction put forward by Allen et al. [44].

All conserved va-miRNAs (denoted as Category-I vamiRNAs) identified in Amur grape had their homologs in 'Summer Black', a hybrid of V. vinifera and V. labrusca, deposited and assigned code No. GEO: GSE24531 [23], with the target genes of these homologous miRNAs have been already reported [27]. We further investigated target genes for only the 34 non-conserved and 72 new miRNAs (denoted as Category-II miRNAs). Typically, most non-conserved miRNAs are species-specific and they are often used to give an insight into their orthologous functions in a few plants, thus making prediction of target genes for non-conserved miRNAs a key preliminary work. In this study, a total of 346 targets for 54 out of all 106 Category-II miRNAs were predicted, with a majority (257) being implicated in a broad range of physiological processes, except for 89 whose functions were unknown (Additional file 7). The number of predicted targets per va-miRNA in Category-II varied drastically from 1 to 103 (Additional file 7). Thirty-four of these va-miRNAs had multiple potential target sites, for instance, va-miR006 which has 103 target sites tops the list, followed by va-miR081 and va-miR003 with 59 and 33 target sites respectively. The other 21 va-miRNAs were predicted to have one target gene each, an indication that they might possess unique functions in regulation of growth and development or response to environmental stress by Amur grape. Besides this, we also observed a trend where multiple members of Category-II va-miRNAs targeted the same single gene and thus had similar functions, but it was not clear whether they co-regulated or independently targeted their target gene spatiotemporally, hence necessitating further investigation. No targets could be predicted for the remaining 52 Category- II miRNAs (Additional file 7), which could be attributed to the limited number of grape EST sequences available in the databases and the difference in grape species used in this work and those used in ESTs sequencing.

To elucidate the functions of Category-II va-miRNAs, target genes with functions annotated for 72 new va-miRNAs were first analyzed and classified into four groups. The first group was involved in stress resistance, for example, disease resistance (NB-ARC, NBSLRR, TIR-NBS-LRR, BZIP, EFR (EF-TU RECEPTOR), RPA1, EDM2, WRKY, GRAM, CBS, IBS1 and TGA1) and abiotic i.e. cold, drought, salt and metal iron stress resistance (HVA22 I, TPR repeat-containing protein, ABRH23, protein RER1B, lactoylglutathione lyase, SPX (SYG1/Pho81/XPR1) domain-containing protein, and aluminum-activated malate transporter). This gives an indication that roles for these potential new miRNAs are comprehensively in the regulation of biotic and abiotic stress responses especially disease, cold and drought resistance, and explains why Amur grape is one of the most important wild grape germplasm with relatively strong stress tolerance. The second group was implicated in several hormone signalling pathways (AUX/IAA, gibberellin, cytokinin, ethylene), calcium, apoptosis, G-protein and membrane protein signals and include the Aux/IAA like protein, two-component response regulator ARR8, AIL6, scarecrow gene regulator, ankyrin repeat family protein, Copine III, TOM (Target of myb1), GTPase, GRAM domain containing protein and integral membrane protein, thus indicating that these new va-miRNAs might participate in important biological pathways. The third group was related to synthesis metabolisms like anthocyanin synthesis (WD40, WD repeat protein, Cytochrome b5 DIF-F and TTG1), sugar (UDP-glucose: glycoprotein, glucose) synthesis (vacuolar invertase 1 (GIN1) (hexose), and rhamnose biosynthetic enzyme 1 (rhamnose)), showing that target genes for this group of new va-miRNAs may be involved in berry coloration and flavor quality formation. The last group of target genes could control flower development, and include FCA gamma, Isoform 2 of AP2-like ethylene-responsive transcription factor AIL6, vernalization independence 4 and scarecrow gene. Interestingly, all the above target genes (LIM, WD, AIL6, GIF1, TGA1.1, WRKY, GRAM, MYB127, 
BZIP, AUX/IAA and PHD) were also important transcript factors or regulator like (Additional file 7). Therefore, these new Amur-specific miRNAs might play far much more significant roles in regulation of growth, development and response to stress of Amur grape.

Among the 34 non-conserved miRNAs, 14 had no target genes, while another 20 were predicted to target 45 genes, out of which 10 had unknown functions. The functions of the other 35 target genes include regulation of metabolic enzymes (Type IIB calcium ATPase, E3 ubiquitin-protein ligase SINAT2, carboxylesterase, oxidoreductase), growth development process (Cellulose synthase D4), stress responses (LRRNT_2 domain containing protein and TPR repeat-containing protein), signal transduction (Type IIB calcium ATPase and WDrepeat membrane protein) and as important transcript factors related to flower development (Transcription factor AP2D23, PHAP2A protein, PHAP2B protein and Squamosa promoter binding protein-homologue 5).
These findings are consistent with those of previous reports on different vitis species [20-24] and other plant species $[11,14-16,34]$.

\section{Verification of potential va-miRNA target genes using $\mathbf{5}^{\prime}$ - RLM-RACE}

To verify the nature of potential miRNA targets and to study how the va-miRNAs regulate their target genes, a modified RLM-RACE experiment was set up. The RLMRACE procedure was successfully used to map the cleavage sites in five predicted va-miRNA target genes. GSVIVT00011815001, GSVIVT00023070001, GSVIVT 00026343001, GSVIVT00025360001 and GSVIVT 00033078001 were confirmed as the real targets of vamiR006, va-miR059, va-miR065, va-miR088, and vamiR104 respectively since all the 5 '-ends in the mRNA fragments mapped to the nucleotide that pairs to the tenth or ninth nucleotide of each miRNA with higher frequencies than depicted for each pairing oligo (Figure 6). All five predicted targets were found to have

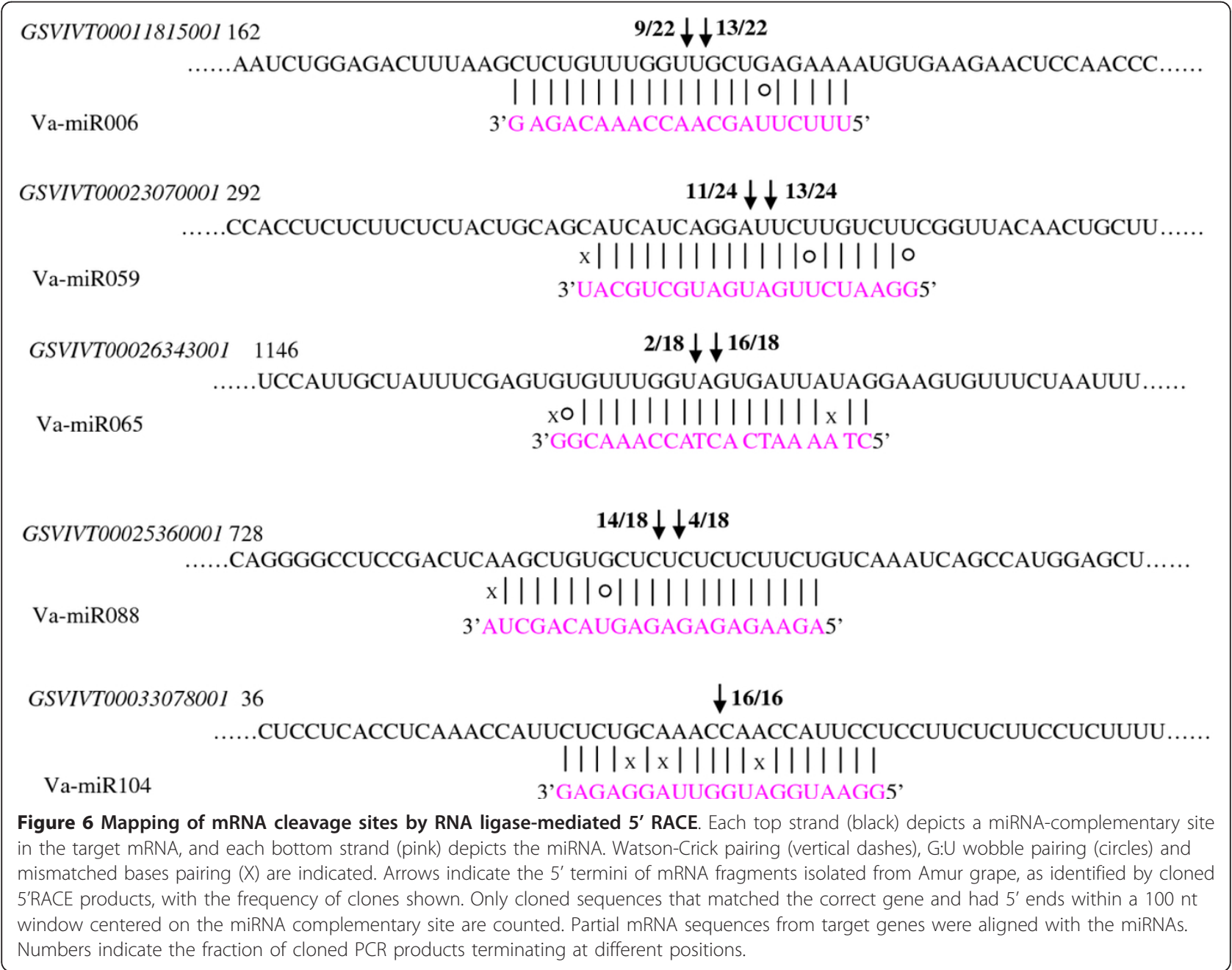


specific cleavage sites corresponding to the miRNA complementary sequences (Figure 6) and might be regulated by the five va-miRNAs. GSVIVT00011815001, GSVIVT00023070001, GSVIVT00026343001, GSVIVT 00025360001, GSVIVT00033078001 are similar to Isoform 2 of AP2-like ethylene-responsive transcription factor AIL6, PHAP2B protein transcription factor, NAD-dependent epimerase/dehydratase, Squamosa promoter binding and Betv I domain containing protein (Additional file 7).

\section{Discussion}

Although several studies on grape miRNAs have recently been reported [20-24], the grape materials used in all the studies reported belong to the cultivated grape Vitis vinifera or hybrids of $V$. vinifera and $V$. labrusca. Amur grape (Vitis amurensis Rupr.) is one of the most important wild species in the grape family. Currently, only a few genes involved in resistance to downy mildew have been identified in Amur grape [45] and there are no known reports on Amur grape miRNAs. The identification of entire sets of miRNAs and their targets will lay a foundation for elucidation of the complex miRNAmediated regulatory systems which control development and other physiological processes $[4,46]$. Systematic studies on miRNAs in Amur grape will contribute to gaining insight into the mechanisms controlling growth and development in this grape species. The advent of deep sequencing technologies has greatly enhanced the capacity of sRNA exploration, and in turn provided a rapid way to identify non-conserved, low accumulation, species-specific as well as conserved miRNAs on a large scale. In this study, using Solexa sequencing, we provide evidence supporting the existence of 106 new and potential, as well as 126 conserved miRNAs in Amur grape. Use of deep sequencing technology, has led to identification of many new grape miRNAs in Vitis vinifera [20,21] or hybrids of $V$. vinifera and $V$. labrusca $[23,24]$, and ITS employment in our study led to the identification of 72 additional miRNAs that are specific to the Amur grape species. Future large scale experimental approaches in more plants are likely to identify additional species-specific miRNAs.

The aim of this work was to identify miRNAs present in Amur grape including miRNAs evolutionary conserved in other plants. Searches for conserved miRNAs revealed that many va-miRNAs have orthologs in other plants like Arabidopsis [28], rice [14], poplar [15], Medicago [47], Solanum lycopersicum Mill [12], peanuts [17], citrus [43], wheat [33] etc. In addition, deep sequencing of the small RNA library allowed for identification of the expression levels of each member of a miRNA family. Sequence analysis reveals that the relative abundance of certain members within the miRNA families varied drastically, from 2 to 372,442 copies. Amongst the conserved miRNAs, va-miR166h topped the list in terms of copy numbers, but the annotation of its function is still not available, consistent with the observation in Summer Black grapevine which is a hybrid of $V$. vinifera and V. labrusca [23,24]. Conversely, va-miR171h, va-miR169i and va-miR159a/b appeared to have only less than 10 copies each (Figure 2). We also discovered that these conserved miRNAs have their orthologs in nearly 30 other plant species/varieties (Table 5), as indicated in previous miRNA reports [48-52]. Some miRNAs are conserved in several plant species, an aspect which will provide an opportunity for assessment of evolution of these families across diverse plants. Seventy two new and potential va-miRNAs were specific to Amur grape and were not detected in other vitis species; while another twenty-four were only conserved in Vitis amurensis Rupr. and Vitis vinifera L. These open the possibility that these miRNA families could have descended from a common ancestor and diverged or were lost during evolution of vitis plants. It is also possible that regulatory interactions directed by these vitis-specific miRNAs are involved in the adaptation to the diverse ecological environments.

Since the roles of miRNAs in development and biotic or abiotic stress regulation are executed through the cleavage or translation repression of target genes, miRNA target prediction is critical for gaining insight to regulatory functions of miRNAs. Before this report, target genes for conserved miRNAs in grape had been systematically investigated in other vitis species $[20,21,23,24,53]$. Conserved miRNAs have high sequence conservation in closely related plant species and thus their target genes also possess some conservation of functions. For non-conserved and new or potential vamiRNAs, we reveal that a number of disease resistance, stress resistance, anthocyanin synthesis regulatory genes as well as some other genes related to secondary metabolism and sugar metabolism might be targeted by vamiRNAs based on previous reports on functional annotation of orthologous genes in other plants [17,41,54-56]. Outstandingly, we found 11 important transcript factors and regulators for va-miRNAs, and these might play a wide range of vital roles during development or response to stress in Amur grape. Other target genes were associated with signal transduction, metabolism, transport, growth and development processes, which is similar to studies by Wu et al. [45] in the Amur grape DNA library. All these observations indicate that both the new and non-conserved va-miRNAs identified in this study might play extensive regulatory roles, not only in development, but also in stress response and diverse physiological processes. 
The availability of a full grape genome and plenty of grape ESTs helped us to identify 72 new, 34 non-conserved va-miRNAs and 346 target genes. It has been reported that target prediction for new miRNAs may yield some false positives [57]. However, blast search using miRNA sequences against EST databases can also be a good way of identifying potential targets of the new miRNAs, followed by necessary experimental verification. In this work, we employed the 5'-RLM-RACE method to detect miRNA-guided cleavage of target mRNAs of five Va-miRNAs, where the results showed that five potential target genes for the five Va-miRNAs had specific cleavage sites corresponding to their miRNA complementary sequences. This confirms the actual existence of these potential va-miRNA target genes. For a thorough investigation of the real existence of all the potential target genes, systematic research should be planned in the next phase of this work. In addition, there is no current information available regarding the functions of more than $1 / 4$ of the target genes identified, which makes it difficult to determine whether these miRNA targets have functional bias. Furthermore, it was also observed that consistent with previous reports [55-57], most va-miRNA targets have a miRNA-complementary site located in their coding regions and occasionally in their 3' or 5'UTRs. Previous reports indicate that plants usually have a lower number of genes targeted by a single miRNA than in animals. For instance, each drosophila miRNA has on average over 50 predicted targets [58], while most Arabidopsis miRNAs have six targets or fewer [4]. In contrast, some va-miRNAs in this study have more target genes than those reported for drosophila and even for Arabidopsis, for example va-miR006 which has 103 target genes, a finding that might imply that Amur grape-specific miRNAs have more extensive functions. This study pioneers the first large scale cloning and characterization of Amur grape miRNAs and their predicted targets thus laying a foundation for future functional studies.

\section{Conclusion}

Deep sequencing of short RNAs from Amur grape flowers and fruits identified 72 new potential miRNAs and 34 known but non-conserved miRNAs, indicating that specific miRNAs exist in Amur grape. These results show that a number of regulatory miRNAs exist in Amur grape and they play an important role in Amur grape growth, development, and response to abiotic or biotic stresses.

\section{Methods}

\section{Plant material}

Leaves, stem $(0.3 \mathrm{~cm}$ in diameter $)$, tendrils, inflorescences, flowers, and developing fruits $(15,30$ and 45 days after full blooming) were collected in 2010 from Amur grape (Vitis amurensis Rupr.) trees grown under general site conditions at the Fruit Germplasm Resources Garden of the Zhengzhou Institute of Pomology, Chinese Academy of Agricultural Sciences, Zhengzhou, China. After collection, all the samples were immediately frozen in liquid nitrogen and stored at $-80^{\circ}$ $C$ until use.

\section{Low molecular RNA extraction}

Total RNA was isolated from $200 \mathrm{mg}$ of the selected plant tissues using the CTAB method [59], then $10 \mathrm{M}$ $\mathrm{LiCl}$ was used to separate the low and larger molecular weight RNA following the procedures reported earlier [17]. The small RNA fraction was then dissolved in 30 $\mu \mathrm{l}$ of RNase free water and the concentration of RNA measured by a UV-1800 spectrophotometer (Shimadzu, Japan) and visually ascertained in a $2.5 \%$ agarose gel. The larger molecular weight RNA samples were used to study the expression patterns of the target genes of vamiRNAs.

\section{Construction and screening of cDNA libraries of small RNAs}

We generated the miRNA-enriched library that has been popularly used to clone miRNAs and to measure the expression of miRNAs via RT-PCR, in which 5'- and 3'end adaptors were linked to the miRNA molecules [36], which were further reverse transcribed using Superscript III reverse transcriptase (Invitrogen) in the presence of random nonamers (Sigma), according to the protocols provided by the manufacturers. After the preparation of miRNA libraries from various organs and tissues, we pooled similar quantities of these library samples for further PCR amplification reactions.

\section{Small RNA sequencing and sequence processing}

The mixed small RNA samples were enriched by polyethylene glycol precipitation, separated on $15 \%$ denaturing PAGE, and visualized by SYBR-gold staining. Small RNAs of 15-30 nt were gel-purified, and then were ligated to a 5'-adaptor and a 3'-adaptor sequentially, which were reversely transcribed to $\mathrm{CDNA}$ with the RT primer (CAAGCAGAAGACGGCATACGA) using Superscript II reverse transcriptase (Invitrogen) [23] for Solexa sequencing and sequence processing by Beijing Genomics Institute (BGI) (Shenzhen, Guangdong, China).

\section{Bioinformatics analysis and identification of va-miRNA and miRNA SNPs}

To identify conserved and potential va-miRNAs in grapevine, the raw sequences were processed as described by Sunkar et al. [55]. The vector sequences of 
all sRNAs sequences from 18 nt to 30 nt were removed, and then the modified sequences were mapped to the grapevine genome, whereby sequences that matched the genome were further subjected to removal of rRNA, tRNA, snRNA, snoRNA and all those containing the polyA tails. The remaining sequences were then compared against known plant miRNAs existing in the miRBase 16.0. Only matching (0-3mismatches) sequences were considered as conserved va-miRNAs, while other sequences that are similar to conserved va-miRNAs, but have one base variation with the conserved va-miRNAs can both be considered one miRNA SNP.

To study potential va-miRNAs precursor sequences, all sRNAs from grapevine were aligned against the grapevine genome and then the miRNA candidates were processed by miRCat http://srna-tools.cmp.uea.ac.uk/ [60], using default parameters, to generate the secondary structures.

\section{Analyses of miRNA by 5'miR-RACE and 3'miR-RACE}

The CDNA was amplified with the mirRacer 5 ' primer (5'- GGACACTGACATGGACTGAAGGAGTA-3') and the mirRacer 3' primer (5'-ATTCTAGAGGCCGAGGCGGCCGACATG-3') to generate a pool of nongene-specific product. 5' miR-RACE reactions were performed with the mirRacer 5' primer and miRNA-genespecific forward primers, and 3' miR-RACE reactions were carried out with the mirRacer 3' primer and miRNA-gene-specific reverse primers (Additional file 8 and Additional file 9), as described by Song et al. [36], with minor modifications. The 5' RACE and 3' RACE clones with PCR products of about $56 \mathrm{bp}$ and $87 \mathrm{bp}$ were sequenced (Invitrogen), respectively.

\section{Prediction of potential target mRNAs for va-miRNAs}

Target predictions were performed based on methods described by Allen et al. [44]. Putative va-miRNAs were first blasted against the grapevine unigene database on the Genoscope http://www.genoscope.cns.fr/. BLASTn hits possessing less than four mismatches were chosen as the candidate targets, and then BLASTx was used to obtain their putative functions.

\section{Data access}

The sRNA sequence data from this study have been submitted to Gene Expression Omnibus (GEO) under accession No. GSE34169 at website: http://www.ncbi. nlm.nih.gov/geo/query/acc.cgi?acc=GSE34169.

\section{Additional material}

Additional file 1: Size distribution of unique small RNA sequences from Amur grape.

Additional file 2: Identified conserved miRNAs from Amur grape.
Additional file 3: Predicted secondary structure of new potential miRNAs from Amur grape.

Additional file 4: 3'RACE and 5' RACE products of Vv-miRNAs amplified by PCR shown in an ethidium bromide-stained agarose gel. Sizes of the molecular weight markers of the bottom and the second bottom bands are $50 \mathrm{bp}$ and $100 \mathrm{bp}$ on $\mathrm{A}, \mathrm{B}, \mathrm{C}$ and $\mathrm{D}$, respectively. Lanes 1-20 are $3^{\prime} R A C E(A)$ and $5^{\prime} R A C E(B)$ products of 20 lower abundance va-miRNAs (va-miR398b, va-miR399c, va-miR828a, vamiR005, va-miR011, va-miR020, va-miR028, va-miR029, va-miR032, vamiR037, va-miR045, va-miR048, va-miR052, va-miR063, va-miR066, vamiR074, va-miR077, va-miR082, va-miR095 and va-miR098, respectively). Lanes 1'-20' are 3'RACE (C) and 5'RACE (D) products of 20 higher abundance va-miRNAs (va-miR156e, va-miR160c, va-miR162, va-miR164c, va-miR166c, va-miR169m, va-miR171c, va-miR172c, va-miR408, vamiR535a, va-miR001, va-miR007, va-miR016, va-miR018, va-miR023, vamiR046, va-miR047, va-miR049, va-miR057 and va-miR062, respectively). The sizes of $3^{\prime} R A C E$ products are about $83 \mathrm{bp}$ while the size of 5'RACE products are about $57 \mathrm{bp}$.

Additional file 5: List of SNPs of conserved miRNAs in Amur grape Additional file 6: List of miR-LDs of conserved miRNAs in Amur grape.

Additional file 7: List of predicted target genes of non-conserved miRNAs identified in Amur grape.

Additional file 8: List of primers of miRNAs used for miR-5'RACE and miR-3'RACE.

Additional file 9: List of primers of va-miR-LDs used for miR-RACE.

\section{Acknowledgements}

This work was supported by the NCET Program of China (Grant No. NCET08-0796), a Project Funded by the Priority Academic Program Development of Jiangsu Higher Education Institutions (PAPD), and the Fundamental Research Funds for the Central Universities (Grant No. KYJ200909).

\section{Author details}

${ }^{1}$ College of Horticulture, Nanjing Agricultural University, 1 Weigang, Nanjing 210095, China. 'Zhengzhou Institute of Pomology, Chinese Academy of Agricultural Sciences, 11 Hanghai East Road, Zhengzhou 450009, People's Republic of China.

\section{Authors' contributions}

WC carried out the laboratory work and wrote this paper. WC and SG performed bioinformatics analyses. HJ and LC participated in coordination of the study. EK and LX constructed the sRNA library. KKN revised this paper. FJ conceived, designed the study and revised this paper. All authors read and approved the final manuscript.

\section{Competing interests}

The authors declare that they have no competing interests.

Received: 15 June 2011 Accepted: 29 March 2012

Published: 29 March 2012

\section{References}

1. Phillips J, Dalmay T, Bartels D: The role of small RNAs in abiotic stress. FEBS Letters 2007, 581:3592-3597.

2. Li Y, Zheng Y, Addo-Quaye C, Zhang L, Saini A, Jagadeeswaran G, Axtell MJ, Zhang W, Sunkar R: Transcriptome-wide identification of microRNA targets in rice. Plant J 2010, 62:742-759.

3. Brodersen $P$, Sakvarelidze-Achard L, Bruun-Rasmussen M, Dunoyer $P$, Yamamoto $Y Y$, Sieburth L, Voinnet O: Widespread translational inhibition by plant miRNAs and siRNAs. Science 2008, 320:1185-1190.

4. Jones-Rhoades MW, Bartel DP, Bartel B: MicroRNAs and their regulatory roles in plants. Annual Review of Plant Biology 2006, 57:19-53.

5. Lauter N, Kampani A, Carlson S, Goebel M, Moose SP: microRNA172 downregulates glossy15 to promote vegetative phase change in maize. Proc Natl Acad Sci USA 2005, 102:9412-9417. 
6. Schwarz S, Grande AV, Bujdoso N, Saedler H, Huijser P: The microRNA regulated SBP-box genes SPL9 and SPL15 control shoot maturation in Arabidopsis. Plant Mol Biol 2008, 67:183-1895.

7. Jung JH, Park CM: MIR166/165 genes exhibit dynamic expression patterns in regulating shoot apical meristem and floral development in Arabidopsis. Planta 2007, 225:1327-1338

8. Ochando I, Jover-Gil S, Ripoll JJ, Candela H, Vera A, Ponce MR, MartínezLaborda A, Micol JL: Mutations in the microRNA complementarity site of the INCURVATA4 gene perturb meristem function and adaxialize lateral organs in Arabidopsis. Plant Physiol 2006, 141:607-619.

9. Borsani O, Zhu J, Verslues PE, Sunkar R, Zhu JK: Endogenous siRNAs derived from a pair of natural cis-antisense transcripts regulate salt tolerance in Arabidopsis. Cell 2005, 123:1279-1291.

10. Lu S, Sun YH, Shi R, Clark C, Li L, Chiang VL: New and mechanical stressresponsive microRNAs in Populus trichocarpa that are absent from Arabidopsis. Plant Cell 2005, 17:2186-2203.

11. Zhang BH, Pan X, Cannon CH, Cobb GP, Anderson TA: Conservation and divergence of plant microRNA genes. Plant J 2006, 46:243-259.

12. Zhao CZ, Xia H, Frazier TP, Yao YY, Bi YP, Li YP, Li AQ, Li MJ, Zhang BH, Wang $X J$ : Deep sequencing identifies new and conserved microRNAs in peanuts (Arachis hypogaea L.). BMC Plant Biol 2010, 10:3.

13. Felippes FF, Schneeberger K, Dezulain T, Huson DH, Weigel D: Evolution of Arabidopsis thaliana microRNAs from random sequences. RNA 2008, 14:2455-2459.

14. Sunkar R, Zhou X, Zheng Y, Zhang W, Zhu JK: Identification of new and candidate $\mathrm{Vv}$-miRNAs in rice by high throughput sequencing. BMC Plant Biol 2008, 8:25

15. Szittya G, Moxon S, Santos DM, Jing R, Fevereiro MP, Moulton V, Dalmay T: High-throughput sequencing of Medicago truncatula short RNAs identifies eight new Vv-miRNA families. BMC Genomics 2008, 9:593.

16. Zhang BH, Pan XP, Stellwag EJ: Identification of soybean microRNAs and their targets. Planta 2008, 229:161-182.

17. Song CN, Fang JG, Li XY, Liu H, Chao TC: Identification and characterization of 27 conserved microRNAs in citrus. Planta 2009, 230:671-685.

18. Song CN, Wang C, Zhang CQ, Nicholas KK, Yu HP, Ma ZQ, Fang JG: Deep sequencing discovery of new and conserved microRNAs in trifoliate orange (Citrus rifoliate). BMC Genomics 2010, 11:431.

19. Yu HP, Song CN, Jia QD, Wang C, Li F, Nicholas KK, Zhang XY, Fang JG: Computational identification of microRNAs in apple expressed sequence tags and validation of their precise sequences by miR-RACE. Physiologia Plantarum 2011, 141:56-70.

20. Pantaleo V, Szittya G, Moxon S, Miozzi L, Moulton V, Dalmay T, Burgyan J: Identification of grapevine microRNAs and their targets using highthroughput sequencing and degradome analysis. The Plant Journal 2010, 62:960-976.

21. Mica E, Piccolo V, Delledonne M, Ferrarini A, Pezzotti M, Casati C, Fabbro CD, Valle G, Policriti A, Morgante M, Pesole G, Horner MpèDS: Correction: high throughput approaches reveal splicing of primary microRNA transcripts and tissue specific expression of mature microRNAs in Vitis vinifera. BMC Genomics 2010, 11:109.

22. Carra A, Mica E, Gambino G, Pindo M, Moser C, Pè ME, Schubert A: Cloning and characterization of small non-coding RNAs from grapevine. Plant $J$ 2009, 59:750-763.

23. Wang C, Wang X, Nicholas KK, Song C, Zhang C, Li X, Han J, Fang J: Deep sequencing of grapevine flower and berry short RNA library for discovery of new microRNAs and validation of precise sequences of grapevine microRNAs deposited in miRBase. Physiologia Plantarum 2011, 143:64-81

24. Wang C, Shangguan LF, Nicholas KK, Wang XC, Han J, Song CN, Fang JG: Characterization of microRNAs identified in a table grapevine cultivar with validation of computationally predicted grapevine miRNAs by miRRACE. PLoSone 2011, 6:e21259.

25. Liang CW, Zhang XW, Zou J, Xu D, Su F, Ye NH: Identification of miRNA from Porphyra yezoensis by High-Throughput Sequencing and Bioinformatics Analysis. PLoSone 2010, 5:e10698.

26. Rajagopalan R, Vaucheret H, Trejo J, Bartel DP: A diverse and evolutionarily fluid set of microRNAs in Arabidopsis thaliana. Genes Development 2006, 20:3407-3425.

27. Dezulian T, Palatnik J, Huson D, Weigel D: Conservation and divergence of microRNA families in plants. Genome Biology 2005, 6:13.
28. Fahlgren N, Howell MD, Kasschau KD, Chapman EJ, Sullivan CM, Cumbie JS, Givan SA, Law TF, Grant SR, Dangl JL, Carringtonet JC: High-throughput sequencing of Arabidopsis micro-RNAs: evidence for frequent birth and death of MiRNA genes. PLoSone 2007, 2:e219.

29. Barakat A, Wall PK, Diloreto S, Depamphilis CW, Carlson JE: Conservation and divergence of microRNAs in populus. BMC Genomic 2007, 8:481.

30. Fahlgren $\mathrm{N}$, Jogdeo $\mathrm{S}$, Kasschau KD, Sullivan CM, Chapman EJ, Laubinger $\mathrm{S}$, Smith LM, Dasenko M, Givan SA, Weigel D, Carrington JC: MicroRNA Gene Evolution in Arabidopsis lyrata and Arabidopsis thaliana. The Plant Cell 2010, 22:1074-1089.

31. Dohm JC, Lottaz C, Borodina T, Himmelbauer H: Substantial biases in ultra-short read data sets from high-throughput DNA sequencing. Nucleic Acids Research 2008, 36:e105.

32. Meyers BC, Axtell MJ, Bartel B, Bartel DP, Baulcombe D, Bowman JL, Cao XF, Carrington JC, Chen XM, Green PJ, Griffiths-Jones S, Jacobsen SE, Mallory AC, Martienssen RA, Poethig RS, Qi YJ, Vaucheret H, Voinnet O, Watanabe $Y$, Weigel D, Zhu JK: Criteria for annotation of plant MicroRNAs. Plant Cell 2008, 20:3186-3190.

33. Yao YY, Guo GG, Ni ZF, Sunkar R, Zu JK, Sun QX: Cloning and characterization of microRNAs from wheat(Triticumaestivum L.). Genome Biology 2007, 8:R96.

34. Zhang BH, Pan XP, Anderson TA: Identification of 188 conserved maize microRNAs and their targets. FEBS Lett 2006, 580:3753-3762.

35. Lu C, Kulkarni K, Souret FF, Muthuvalliappan R, Tej SS, Poethig RS, Henderson IR, Jacobsen SE, Wang WZ, Green PJ, Meyers BC: MicroRNAs and other small RNAs enriched in the Arabidopsis RNA-dependent RNA polymerase-2 mutant. Genome Res 2006, 16:1276-1288.

36. Song CN, Fang JG, Wang C, Guo L, Nicholas KK, Ma ZQ: MiR-RACE, a new efficient approach to determine the precise sequences of computationally identified trifoliate orange (Poncirus rifoliate) MicroRNAs. PLoSone 2010, 5:e10861.

37. Sun GH, Yan J, Noltner K, Feng J, Li HT, Sardis DA, Sommer SS, Rossi JJ: SNPs in human miRNA genes affect biogenesis and function. RNA 2009, 15:1640-1651.

38. Liu D, Fan J, Mei MX, Ingvarsson S, Chen HP: Identification of miRNAs in a liver of a human fetus by a modified method. PLoSone 2009, 4:e7594.

39. Ehrenreich IM, Purugganan MD: Sequence variation of MicroRNAs and their binding sites in Arabidopsis. Plant Physiology 2008, 146:1974-1982.

40. Lai EC, Tomancak P, Williams RW, Rubin GM: Computational identification of Drosophila microRNA genes. Genome Biol 2003, 4:R42.

41. Bonnet $E$, Wuyts J, Rouze P, Peer VY: Detection of 91 potential in plant conserved plant microRNAs in Arabidopsis thaliana and Oryza sativa identifies important target genes. Proc Natl Acad Sci USA 2004, 101:11511-11516.

42. Rhoades MW, Reinhart BJ, Lim LP, Burge CB, Bartel B, Bartel DP: Prediction of plant microRNA targets. Cell 2002, 110:513-520.

43. Song CN, Jia QD, Fang JG, Li F, Wang C, Zhang Z: Computational identification of citrus microRNAs and target analysis in citrus expressed sequence tags. Plant Biology 2009, 12:927-934.

44. Allen E, Xie Z, Gustafson AM, Carrington JC: MicroRNA-directed phasing during trans-acting siRNA biogenesis in plants. Cell 2005, 12:207-221.

45. Wu J, Zhang $Y l$, Zhang HQ, Huang H, Folta KM, Lu J: Whole genome wide expression profiles of Vitis amurensis grape responding to downy mildew by using Solexa sequencing technology. BMC Plant Biology 2010, $10: 234$.

46. Zhan $\mathrm{BH}$, Wang QL, Pan XP: MicroRNAs and their regulatory roles in animals and plants. Journal of Cellular Physiology 2007, 210:279-289.

47. Zhang J, Zeng R, Chen J, Liu X, Liao Q: Identification of conserved microRNAs and their targets from Solanum lycopersicum Mill. Gene 2008, 423:1-7.

48. Floyd SK, Bowman JL: Gene regulation: ancient micro-RNA target sequences in plants. Nature 2004, 428:485-486.

49. Floyd SK, Zalewski CS, Bowman JL: Evolution of class III homeodomain leucine zipper genes in streptophytes. Genetics 2006, 173:373-388.

50. Arazi T, Talmor-Neiman M, Stav R, Riese M, Huijser P, Baulcombe DC: Cloning and characterization of micro-RNAs from moss. Plant Journal 2005, 43:837-848.

51. Axtell MJ, Bartel DP: Antiquity of microRNAs and their targets in land plants. Plant Cell 2005, 17:1658-1673.

52. Fattash I, Voss B, Reski R, Hess WR, Frank W: Evidence for the rapid expansion of microRNA-mediated regulation in early land plant evolution. BMC Plant Biology 2007, 7:13 
53. Jaillon O, Aury JM, Noel B, Policriti A, Clepet C, Casagrande A, Choisne N, Aubourg S, Vitulo N, Jubin C, Vezzi A, Legeai F, Hugueney P, Dasilva C, Horner D, Mica E, Jublot D, Poulain J, Bruyère C, Billault A, Segurens B, Gouyvenoux M, Ugarte E, Cattonaro F, Anthouard V, Vico V, Fabbro CD, Alaux M, Gaspero GD, Dumas V, Felice N, Paillard S, Juman I, Moroldo M, Scalabrin S, Canaguier A, Clainche IL, Malacrida G, Durand E, Pesole G, Laucou V, Chatelet P, Merdinoglu D, Delledonne M, Pezzotti M, Lecharny A, Scarpelli C, Artiguenave PèG, Valle F ME, Morgante M, Caboche M, AdamBlondon AF, Weissenbach J, Quétier F, Wincker P: French-Italian Public Consortium for Grapevine Genome Characterization: The grapevine genome sequence suggests ancestral hexaploidization in major angiosperm phyla. Nature 2007, 449:463-467.

54. Jones-Rhoades MW, Bartel DP: Computational identification of plant microRNAs and their targets, including a stress induced miRNA. Molecular Cell 2004, 14:787-799.

55. Sunkar R, Girke T, Jain PK, Zhu JK: Cloning and characterization of microRNAs from rice. Plant Cell 2005, 17:1397-1411.

56. Sunkar R, Girke T, Zhu JK: Identification and characterization of endogenous small interfering RNAs from Rice. Nucleic Acids Research 2005, 33:4443-4454.

57. Moxon S, Jing RC, Szittya G, Schwach F, Rusholme Pilcher RL, Moulton V, Dalmay T: Deep sequencing of tomato short RNAs identifies microRNAs targeting genes involved in fruit ripening. Genome Research 2008, 18:1602-1609.

58. Grun D, Wang YL, Langenberger D, Gunsalus KC, Rajewsky N: microRNA target predictions across seven Drosophila species and comparison to mammalian targets. PloS Comput Biol 2005, 1:e13.

59. Chang $S$ J, Jepf $P$, Johnc A: Simple and efficient method for isolating RNA from pine trees. Plant Mol Biol Reporter 1993, 11:113-116.

60. Moxon S, Schwach F, Maclean D, Dalmay T, Studholme DJ, Moulton V: A tool kit for analyzing large-scale plant small RNA datasets. Bioinformatics 2008, 24:2252-2253.

doi:10.1186/1471-2164-13-122

Cite this article as: Wang et al: Identification of microRNAs from Amur grape (vitis amurensis Rupr.) by deep sequencing and analysis of microRNA variations with bioinformatics. BMC Genomics 2012 13:122.

\section{Submit your next manuscript to BioMed Central and take full advantage of:}

- Convenient online submission

- Thorough peer review

- No space constraints or color figure charges

- Immediate publication on acceptance

- Inclusion in PubMed, CAS, Scopus and Google Scholar

- Research which is freely available for redistribution

Submit your manuscript at www.biomedcentral.com/submit
Biomed Central 